\title{
Swarm Intelligence and Cyber-Physical Systems: Concepts, Challenges and Future Trends
}

DOI:

10.1016/j.swevo.2020.100762

https://doi.org/10.1016/j.swevo.2020.100762

\section{Document Version}

Accepted author manuscript

Link to publication record in Manchester Research Explorer

\section{Citation for published version (APA):}

Schranz, M., Di Caro, G., Schmickl, T., Elmenreich, W., Arvin, F., Sekercioglu, A., \& Sende, M. (2021). Swarm Intelligence and Cyber-Physical Systems: Concepts, Challenges and Future Trends. Swarm and Evolutionary Computation, 60, [100762]. https://doi.org/10.1016/j.swevo.2020.100762,

https://doi.org/10.1016/j.swevo.2020.100762

Published in:

Swarm and Evolutionary Computation

\section{Citing this paper}

Please note that where the full-text provided on Manchester Research Explorer is the Author Accepted Manuscript or Proof version this may differ from the final Published version. If citing, it is advised that you check and use the publisher's definitive version.

\section{General rights}

Copyright and moral rights for the publications made accessible in the Research Explorer are retained by the authors and/or other copyright owners and it is a condition of accessing publications that users recognise and abide by the legal requirements associated with these rights.

\section{Takedown policy}

If you believe that this document breaches copyright please refer to the University of Manchester's Takedown Procedures [http://man.ac.uk/04Y6Bo] or contact uml.scholarlycommunications@manchester.ac.uk providing relevant details, so we can investigate your claim.

\section{OPEN ACCESS}




\section{Journal Pre-proof}

Swarm Intelligence and Cyber-Physical Systems: Concepts, Challenges and Future Trends

Melanie Schranz, Gianni A. Di Caro, Thomas Schmickl, Wilfried Elmenreich, Farshad Arvin, Ahmet Şekercioğlu, Micha Sende

PII:

DOI:

Reference:

To appear in:

Received date:

Revised date:

Accepted date:
S2210-6502(20)30415-6

https://doi.org/10.1016/j.swevo.2020.100762

SWEVO 100762

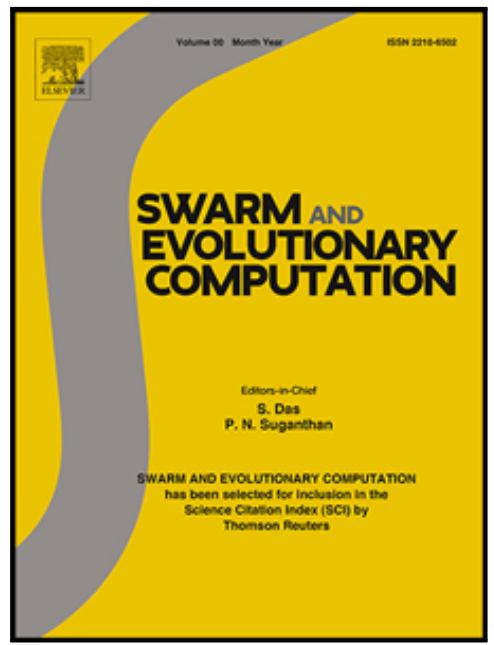

Please cite this article as: Melanie Schranz, Gianni A. Di Caro, Thomas Schmickl, Wilfried Elmenreich, Farshad Arvin, Ahmet Şekercioğlu, Micha Sende, Swarm Intelligence and Cyber-Physical Systems: Concepts, Challenges and Future Trends, Swarm and Evolutionary Computation (2020), doi: https://doi.org/10.1016/j.swevo.2020.100762

This is a PDF file of an article that has undergone enhancements after acceptance, such as the addition of a cover page and metadata, and formatting for readability, but it is not yet the definitive version of record. This version will undergo additional copyediting, typesetting and review before it is published in its final form, but we are providing this version to give early visibility of the article. Please note that, during the production process, errors may be discovered which could affect the content, and all legal disclaimers that apply to the journal pertain.

(C) 2020 Published by Elsevier B.V. 


\title{
Swarm Intelligence and Cyber-Physical Systems: Concepts, Challenges and Future Trends
}

Melanie Schranz ${ }^{\mathrm{a}}$, Gianni A. Di Caro ${ }^{\mathrm{b}}$, Thomas Schmickl ${ }^{\mathrm{c}}$, Wilfried Elmenreich ${ }^{\mathrm{d}}$, Farshad Arvin ${ }^{\mathrm{e}}$, Ahmet Şekercioğlu ${ }^{\mathrm{f}}$, Micha Sende ${ }^{\mathrm{a}}$

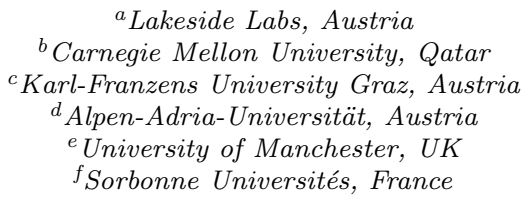

${ }^{a}$ Lakeside Labs, Austria

${ }^{b}$ Carnegie Mellon University, Qatar

${ }^{c}$ Karl-Franzens University Graz, Austria

${ }^{d}$ Alpen-Adria-Universität, Austria

${ }^{e}$ University of Manchester, UK

${ }_{\text {Sorbonne Universités, France }}$

\begin{abstract}
Swarm Intelligence (SI) is a popular multi-agent framework that has been originally inspired by swarm behaviors observed in natural systems, such as ant and bee colonies. In a system designed after swarm intelligence, each agent acts autonomously, reacts on dynamic inputs, and, implicitly or explicitly, works collaboratively with other swarm members without a central control. The system as a whole is expected to exhibit global patterns and behaviors. Although well-designed swarms can show advantages in adaptability, robustness, and scalability, it must be noted that SI system havent really found their way from lab demonstrations to real-world applications, so far. This is particularly true for embodied SI, where the agents are physical entities, such as in swarm robotics scenarios.

In this paper, we start from these observations, outline different definitions and characterizations, and then discuss present challenges in the perspective of future use of swarm intelligence. These include application ideas, research topics, and new sources of inspiration from biology, physics, and human cog-

Email addresses: schranz@lakeside-labs.com (Melanie Schranz), gdicaro@cmu.edu (Gianni A. Di Caro), thomas.schmickl@uni-graz.at (Thomas Schmickl),

wilfried.elmenreich@aau.at (Wilfried Elmenreich), farshad.arvin@manchester.ac.uk (Farshad Arvin), ahmet@sekerci.info (Ahmet Şekercioğlu), sende@lakeside-labs.com (Micha Sende)
\end{abstract}


nition. To motivate future applications of swarms, we make use of the notion of cyber-physical systems (CPS). CPSs are a way to encompass the large spectrum of technologies including robotics, internet of things (IoT), Systems on Chip (SoC), embedded systems, and so on. Thereby, we give concrete examples for visionary applications and their challenges representing the physical embodiment of swarm intelligence in autonomous driving and smart traffic, emergency response, environmental monitoring, electric energy grids, space missions, medical applications, and human networks. We do not aim to provide new solutions for the swarm intelligence or CPS community, but rather build a bridge between these two communities. This allows us to view the research problems of swarm intelligence from a broader perspective and motivate future research activities in modeling, design, validation/verification, and human-in-the-loop concepts.

Keywords: Swarm intelligence, Cyber-physical systems, Swarm robotics

\section{Introduction}

The trend in today's technologies is that computers are becoming embedded in everyday objects. Such systems range from Systems on Chip (SoC) that make the computational core of modern everyday devices such as smartphones, 5 to the Internet of Things (IoT) that connects billions of edge devices, and the Internet of Everything (IoE), which brings together people, processes, data, and devices. In short, we can say that the world is becoming truly collective and connected, increasingly featuring systems that integrate large numbers of multiple interacting components at different scales. Nevertheless, the era of pervasive computing is still at the very beginning, and a number of fundamental issues related to design, computation, prediction, and control in large systems of interacting autonomous components still need to be addressed.

In this respect, Cyber-physical systems (CPSs) have recently emerged as the leading domain for the study and implementation of multi-component systems where computational and physical resources are strictly interconnected [1]. The National Science Foundation precisely defines CPSs as "Engineered systems 
that are built from, and depend upon, the seamless integration of computation and physical components.". They integrate sensing, computation, control, and networking into physical objects and infrastructure, and let them interact [2]. CPSs represent a major paradigm in the framework of collective and connected systems, as well as a vertical study of systems: they are inherently transdisciplinary, generalizing and expanding individual sub-fields such as embedded systems, robotics, and networking, by simultaneously merging concepts from cybernetics, mechatronics, design, and process science [3].

The CPS model is hence well-suited to describe and reason on the plethora of complex mechatronical components that are being to be pervasively deployed, connected, and integrated with our social networks and our lifes in general, creating what can be termed a cyber-physical-socialsystems [4]. Indeed, each potentially complex component of these large systems is a CPS. Such that their 30 multi-scale interconnection and interaction is better captured by the notion of Cyber-Physical System of Systems (CPSoS), defining a large and distributed complex system of CPSs. CPSoS are a specific (yet very broad) instance of a System of Systems (SoS) [5]: an ecosystem of CPSs.

It is well understood that aspects related to decisional autonomy, system integration, cyber-security, control, scalability, optimization, validation, and verification, all play a major role in CPSs' design and control. Moreover, CPSs are meant to operate in the physical world, which typically faces continual dynamic changes, features events and external conditions that are hard to predict or model, include other CPSs as well as human agents. The presence of all these aspects to account for, as well as the fact that a CPS is the product of integration of heterogeneous systems, pose several open research challenges [6].

If we escalate to the more general CPSoS model, the design and control challenges become even harder, since individual component autonomy must be glued by explicitly addressing interdependence and coordination, interoperability, dis45 tributed control and emergence of behaviors (e.g., [5], Table IV). In general, centralized and static management and control model are not expected to be the right solution approach to address all these challenges. Instead, the methods 
that should be employed should achieve the following goals:

- distributed control, supervision and management,

- local coordination among the composing subsystems,

- partial autonomy of the subsystems,

- capability of dynamic reconfiguration of the system as a whole on different time-scales,

- evolution of the overall system during its operation,

- possibility of generating useful emerging behaviors at the system level [7].

No single framework or recipe can obviously tackle all these different and multi-disciplinary challenges. In this case, even solution approaches need to be based on a systems-of-solutions! Summaries of approaches for different domains can be found for instance in $[6,8,9,10$

Among the approaches that can potentially be a good match to tackle the numerous challenges of CPS/CPSoS, ${ }^{1}$ the framework of swarm intelligence [11] (SI) is expected to play a prominent role. In fact, it can be immediately noted that the above given characterizations for CPSs naturally match the concept of a swarm system, and the above list of goals overlaps quite well with the typical 65 features that are expected to be found in a system designed after SI.

SI builds on concepts of self-organized coordination and control in natural swarm systems, such as insect colonies that live in extremely diverse and dynamic environments. An engineered swarm system consists of a population of autonomous agents/components that might range from being relatively sim70 ple (e.g., artificial ants) to quite complex (e.g., self-driving cars), depending on the scenario and on computational or money budgets. The agents reach a

\footnotetext{
${ }^{1}$ Given that a CPSoS is a CPS itself, in the following, we will use the acronym CPSs to refer in a general sense to either a single CPS or to a system of CPSs if the distinction is not semantically relevant in the given context.
} 
common global goal collaboratively using relative simple rules locally. If rules are well-designed, a collective behavior that can solve complex tasks emerges through these interactions with each other and/or with the environment. Typical properties of a system designed after SI shall include parallel and distributed processing and control, locality of interactions, scalability of performance, adaptation to dynamic variations, and resilience to losses and individual component failures.

This paper precisely revolves about the equivalence between CPSs and SI to gain a better understanding of how SI can fruitfully be used to tackle the core challenges presented by CPSs.

Applying SI concepts to CPSs is not an entirely new idea. For instance, if we look at swarm robotics it is apparent that a lot of work exists in this respect, with notable progress in the recent years $[12,13]$. However, applying SI to more general CPSs, and more specifically to real-world CPSs of the kind of the large, heterogeneous, multi-scale systems discussed above, it is clear that several issues still need to be addressed. In particular, two fundamental problems can be readily identified. First, the plethora of different existing CPSs needs a formalization from the perspective of SI. This includes deriving properties and parameters common among different CPSs, which are relevant for the design of SI behavior. Second, the domain of SI has still many open research topics itself. They need to be classified and analyzed for their relevance to CPSs. To summarize, there is the compelling need to clearly identify challenges and research directions of controlling CPSs using SI.

In this paper, we contribute to solve this challenge by reviewing the SI approach from a CPS perspective and reviewing the challenges in CPS design when applying methodologies from SI. The bulk of work in swarm robotics provides a good starting point in this respect since it integrates multiple physical agents and computations. We first characterize properties of CPSs that are relevant for the design of SI algorithms and provide definitions of swarms of CPSs. Based on that we highlight challenges when applying SI and give an outlook for future inspirations, visionary applications, and open research challenges. 
We do not aim to provide new solutions to research problems of the SI or CPS community but rather build a bridge between these two communities. This allows us to view the research problems of SI from a broader perspective and motivate future research activities. This is highly relevant as in future there will be more and more connected CPSs, which will be hard to design, implement, and deploy with traditional approaches. Therefore, we require new approaches that are applicable in general and do not require the control of individual agents but rather let them be self-organized.

The paper has following structure: In Section 2 SI definitions and characterizations are described in the context of CPS swarms. This includes properties and inspirational sources of SI as well as CPS properties. Section 3 summarizes challenges when using SI and discusses why SI algorithms are comparably 115 seldom used in real-world applications. Section 4 gives a broad overview of future work in terms of potential inspirations for SI algorithms and visionary applications for swarms of CPSs. The main contribution is to provide new ideas and prospects for applications in the different domains of CPS swarms and additionally to highlight their limitations and opportunities. Finally, Section 5 summarizes the paper and motivates researchers for future research work.

\section{Definitions and Characterizations}

Even though SI algorithms have started to appear in the research literature relatively recently, according to the Google's Ngram viewer ${ }^{2}$ the term swarm was quite popular until the 1960s. It has then fallen out of fashion for a while and made a strong comeback after year 2000. The early definition of a swarm however, was rather simple, only referring to a group of animals moving together. This is still the basic definition of the Oxford Dictionary, where a swarm is " $A$ large or dense group of flying insects". The term swarm intelligence (SI) was first introduced in 1989 by Beni and Wang [14] in order to describe the dynamics

\footnotetext{
${ }^{2}$ https://books .google.com/ngrams/graph? content=swarm
} 

effective solutions. The quest of SI is to understand conditions, rules, and interactions patterns that can prime swarm behaviors. In turn, this understanding can be used to shed a light on how swarming happens in natural systems and to design novel artificial systems exhibiting effective swarm behaviors.

In this paper we do not try to give yet-another-definition of SI, but we rather build on existing ones to discuss additional relevant aspects for the characterization and verification of SI properties. Bonabeau and Meyer [16] effectively summarize the characteristics of natural swarm systems to provide the following definition of SI: "Social insects work without supervision. In fact, their teractions among individuals in the colony. Although these interactions might be primitive (one ant merely following the trail left by another, for instance), taken together they result in efficient solutions to difficult problems (such as finding the shortest route to a food source among myriad possible paths). The collective

of a group of cellular robots that could be framed as a form of intelligent collective behavior. This marks the point at which swarm behaviors were started to be studied outside of natural sciences, although animal behavior has always continued to be a major source of inspiration for SI developments.

In the animal kingdom, nature has evolved different swarm behaviors as a way for the different organisms to collaboratively reach common global goals like foraging, nest-building, or fighting enemies. The animals in the group coordinate their actions based on their sensory-motor characteristics, giving raise to swarm behaviors such herding, shoaling, schooling, and flocking. SI models build on the observation of natural systems to gain an understanding of the observed in animal systems that although the goal of the group can be rather complex, each individual swarm member follows a set of relatively simple rules, and locally interacts with other individuals and with the environment. Typically, a single swarm member is not capable of finding an efficient solution by itself [15], but under the appropriate conditions the swarm is capable of finding 
ligence." This representation of collective behaviors has been at the core of the design of a number of successful SI algorithms for optimization, networking, and decision-making problems. The Particle Swarm Optimization (PSO) [17] and Ant Colony Optimization (ACO) [18] meta-heuristics are probably the most popular SI frameworks in this respect, where the agent is a minimalist unit, designed after the properties of an individual animal in a natural swarm of reference (e.g., flocking birds for PSO, ant colonies for ACO). Usually, agents are abstracted software agents. The extension of the same concepts to embodied systems was started with the field of swarm robotics, which is well captured by the definitions of Şahin and Spears [19]: "Swarm robotics is the study of how a swarm of relatively simple physically embodied agents can be constructed to collectively accomplish tasks that are beyond the capabilities of a single one" and "Swarm robotics emphasizes self-organization and emergence, while keeping in mind the issues of scalability and robustness."

\subsection{Properties and Fingerprints of a Swarm}

Typical principles of SI were defined in literature several times, the most specific definition was most likely coined by Marc Millonas [20], defining specific requirements of how SI algorithms should operate. The recurrent idea is that they should be able to produce complex and scalable group behavior from simple and local rules/computations. In nature, the behavior of social animals appears to be adaptive, robust, and scalable (see Figure 1) [21]. These are desirable properties of a system that SI design precisely aims to replicate in technical systems. Adaptability represents the ability of a swarm to adapt to dynamic environments, and to cope with different tasks. By exploiting robustness, a swarm can cope with disturbances and failures, such as the loss or the malfunctioning of individual agents. Scalability gives a swarm the ability to perform well with different numbers of swarm members and with differently sized problems. Adding or removing swarm members does not lead to a significant decline in performance, as long as their number does not fall below a critical 

verifiable swarm behavior including all characteristics mentioned above.

Adaptability
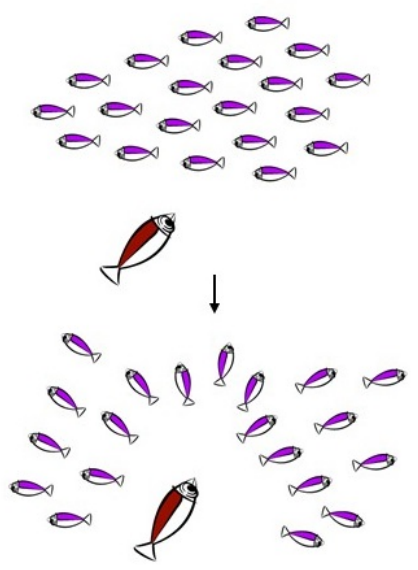

Robustness

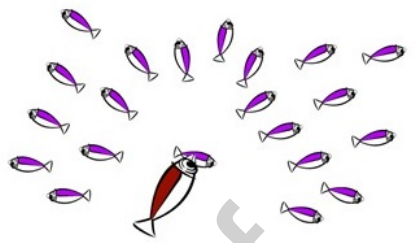

Scalability

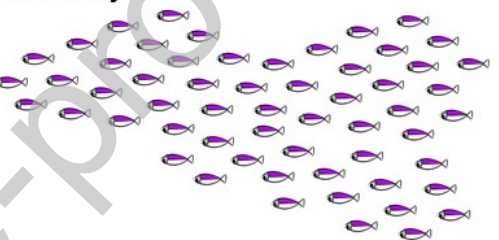

Figure 1: Swarm advantages adaptability, robustness, and scalability (adapted from [22]).

In addition to these characteristics that are already well understood to be the fingerprints of true swarm behavior, two additional crucial aspects should be noted as well: Critical mass and super-linear scaling of the system performance are two system features that can be seen as mandatory for a true swarm system and have been discussed in current literature only marginally.

Critical mass: The advantages of SI algorithms can only be exploited if a critical mass of swarm members is reached. In natural swarm systems, such as honeybees' or cockroaches' collective aggregation decisions [23, 24] as well as in autonomous robotic swarms [25], it was found that the number or density of agents plays an important role for the swarm performance and that swarm systems below a critical number of agents do not function well as a collective.

\footnotetext{
${ }^{3}$ It should be noted that it is not yet clarified what this critical threshold (the minimum number of swarm members) should be [12].
} 
Such a critical mass threshold can be illustrated by the example of the formation process of a sand dune: Given three grains of sand, together they do not form a sand dune although they have to obey to the same physical laws as the billions of sand grains that form a massive dune. This is because the order-generating feedback loops do not impart any effect strongly enough to overcome the system's noise that drives it towards the disorder. In general, SI applications do not work well below a critical mass, but increasingly well above this threshold up to a size where other effects reduce swarm performance again.

Super-linear performance scaling: When scaling up beyond the critical mass, it is expected that in any collective system a large group of agents will achieve more work in total than a smaller group in the same time. In a true swarm, the interactions between the swarm members should exhibit super-linear characteristics, i.e., the effect of the overall system is required to be more than the sum of the effects of its individual parts. Examples are described for honeybees [23], for robotic swarms [26, 27], and for multi-processor systems [28]. Only if the synergies of cooperation boost each individual swarm members' performance, the overall system is a well designed swarm application. This can only be achieved when the local control algorithms of the swarm members are well-designed for the problem. In fact, this means that within the bounds of feasible swarm sizes, not only the efficiency of the whole swarm as a group, but also the efficiency of each single individual has to increase. We refer to this as swarm effect,

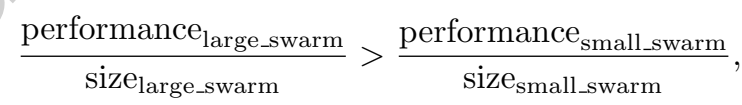

where performance ${ }_{i}$ may represent any quantitative performance metric of of the swarm, and size $_{i}$ indicates the size of that swarm, given by the number of swarm members.

Figure 2 illustrates the expected performance scaling properties of three different systems:

(a) The performance of a hypothetical perfectly scaling disembodied algo- 
rithm or a swarm model that does not care for physical constraints at all, which will scale linear with $O(n)$ as the dashed line in Figure 2, where $\mathrm{n}$ is the number of swarm members.

(b) The performance scaling of an algorithm that shares resources with other algorithm instances or a swarm model that considers space as a shared resource for agents, which will scale $O(\log n)$ as the dotted line in Figure 2 .

(c) The performance of a physically embodied swarm system operating in the real world as the solid hat-shaped curve in Figure 2.

The inequality of Equation 1 holds only for swarms of a size between the threshold values $C_{1}$ and $C_{3}$ as indicated by the dashed area in Figure 2. Thus, it is important to define the number of agents in a swarm in a way that the final performance falls within this parameter-space region of the swarm effect

With very low swarm size or density $N<C_{1}$, the depicted swarm shows almost no performance at all, as the connectivity between the agents is not high enough to allow them to perform their cooperative work at a significant level. As the swarm gets larger, the swarm performs better and better and better as the swarm effect starts to kick in and the swarm increases its performance quickly. At a swarm size of $N>C_{1}$ the swarm can exploit the synergies between swarm members to an extent that it starts to scale in a super-linear way with swarm size. It can keep this extraordinary performance level for a given size range of $C_{1}>N \geq C_{3}$ and keep its performance still above a logarithmic scale for all $N<C_{4}$. The overall peak performance of the total swarm system is exceeded at a threshold of $C_{2}$, in consequence any further swarm member added to the swarm beyond this size starts already to decrease the average performance of each member due to crowding or similar issues. As soon as the swarm size exceeds another critical level $N>C_{4}$ the swarm performance deteriorates converging towards a total loss of performance. We think that these are typical features of swarm systems as soon as they operate in a physically embodied way.

Many SI algorithms rely on mathematical models, for example ordinary differential equations (ODE) or partial differential equations (PDE) models. Such 


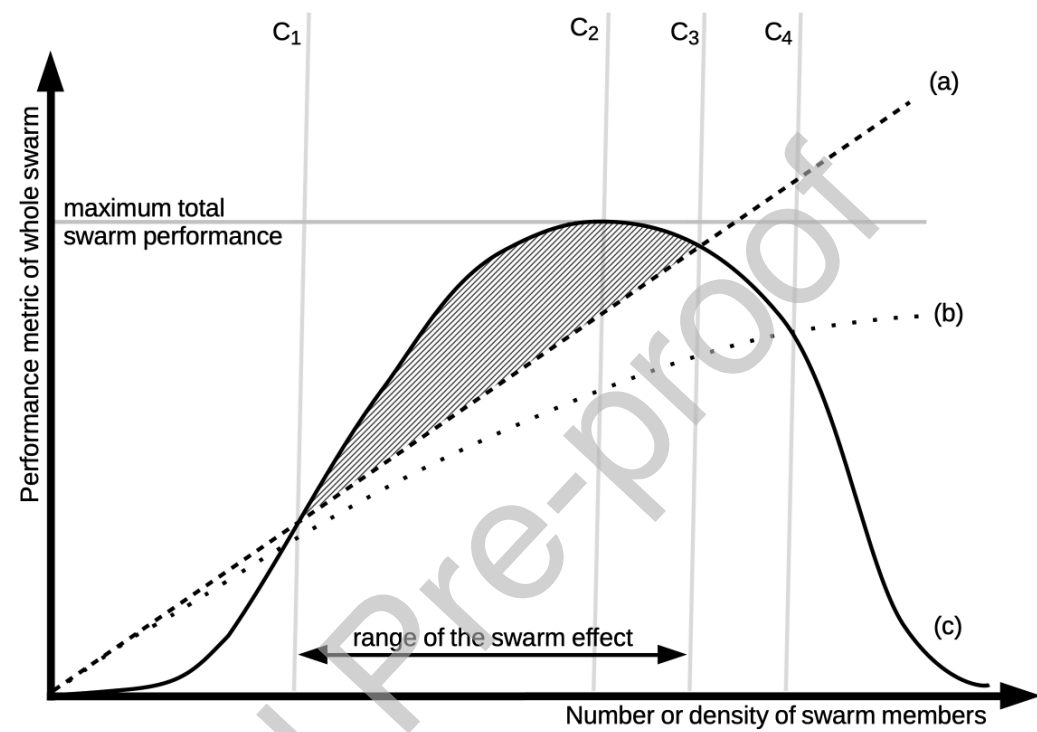

Figure 2: This Figure illustrates the scaling principles discussed here. Only within a swarm size (or density) within the dashed area a swarm effect can be observed, in which each individual swarm member delivers a higher work performed than it would do if working alone or when working in parallel in a purely linear scaling way with other instances. Dashed line (a): Hypothetical of a disembodied algorithm that would scale linearly with swarm size. Dotted line (b): Logarithmic scaling of a disembodied algorithm that requires shared resources amongst its instances when implemented for a parallel execution. Solid line (c): Typical scaling of physically embodied swarms (or of physics-considering simulation of such agents), generalized from several examples given in literature [27, 12], as well as considerations explained in detail in the main text. 
models necessarily abstract many aspects of the physical, chemical and biological system they depict. This can be a critical issue when it comes to physical implementations. Thus, it may happen that a swarm is predicted to be perfectly working when modeled as an ODE or PDE, but this happen differently when the model is tested in the real world. The problem is that, e.g., ODE models by definition implicitly assume that all swarm members are infinitesimally small points in space, thus they cannot crowd or even jam the collective motion. In such models, members are assumed to be always uniformly distributed in space so that interactions are possible among all swarm members in all moments of time at a non-zero extent. However, in a real swarm systems none of these assumptions really hold, some are even explicitly unwanted as, e.g., the agent-to-agent interaction is often on purpose restricted to local neighbors. Of course, we can construct ODE models in a way to respect some of the properties of physically embodied swarms. However, this requires extra treatment and makes these models immediately significantly more complex, and therefore less effective for predictions. One analysis of such an approach can be seen in [29], describing several model-building steps for a very simple swarm algorithm: the BEECLUST algorithm. This algorithm is derived from honeybee experiments that studied natural collective decision-making [23] and was in consequence implemented in a robot swarm [30]. Spatial aspects concerning locality of interaction have to be considered while building the model and the models have to explicitly account for overcrowding issues in order to achieve predictions that compare well to experiments with physically embodied agents (robots, living honeybees). Indeed, this usually requires the use of PDE models, or even stochastic PDEs, which are inherently more problematic to deal with compared to ODEs.

In general terms, abstractions towards simple models can become quickly useless when it comes to real-world implementation of CPS swarms. Algorithms that are predicted to scale perfectly in simple models might easily fall prey to overcrowding, blocking, or traffic jams. This can lead to a situation where the swarm members spend much more time in collision prevention and jam- 
avoiding procedures than in the actual SI algorithm procedures, and therefore not scaling at all. This happened, for example, when the perfectly working ODE-based model of a system based on ant pheromone trails was ported into a robotic system that tried to exploit stigmergic cues in the real world [31].

Scaling properties are indeed an essential fingerprint of a swarm behavior. For being called swarm-intelligent, an algorithm should work better and better, the larger the swarm gets, within feasible swarm size bounds. This was shown for instance by Bodi et al. [27] with the SI algorithm BEECLUST [32].

We suggest here the following simple test consisting of two questions to classify whether a system is swarm-intelligent or not. A good comparison of empiric data suggesting such a test can be found in [12]

1. Does it work even if only one swarm member acts alone? If the system shows the same capabilities even when it consists of only one active agent, then these capabilities do not arise from any swarm behavior, even if many of such agents are operating in parallel.

2. Does the ratio of overall performance to number of swarm members decrease with larger swarm sizes? If the average performance per member goes down with increasing swarm size across the whole size spectrum then this system has obyiously problems with scaling up, instead of drawing an advantage from larger population sizes.

If, and only if, both questions are answered with $N o$, the system should be considered to be truly swarm-intelligent. In particular, these aspects should hold for gradually increasing the swarm size from one up to a feasible amount of swarm members and not up to infinity because every swarm system has some physical limitations.

\subsection{Sources of Inspiration}

Originally, SI algorithms were considered as a subset of bio-inspired algorithms, while these are considered as a subset of nature-inspired algorithms [33]:

swarm intelligence $\subset$ bio-inspired $\subset$ nature-inspired, 
This makes sense as those natural systems that are identified as swarms have exhibit SI properties. However, the bio-inspired source of inspiration is not a strict line of separation, as today the field seems open towards multiple sources of inspiration. Many SI algorithms have been published recently that are not strictly inspired from natural systems, but from other processes that may still and its variants [34], the Swarm Chemistry Particle algorithms [35], and various variants of the Harmony Search algorithm [36], that show typical SI properties but lack a concrete biological source of inspiration for their microscopic rule set.

While many algorithms without a biological inspiration are being labeled as SI, it is worth noticing that a number of approaches that indeed are bio-inspired, population-based, and aim to produce system-level outputs, are usually not classified as SI. For example, this is the case of evolutionary computation, selforganizing neural networks, and cellular automata. The reason might be related to the fact that SI was historically restricted to be the domain of the algorithms inspired by swarm behaviors in animal groups.

Other frameworks that are inspired by natural processes but are not SI, include biomimetic algorithms, that are based on the imitation of biological processes and models, and as such can totally depart from the ideas of SI, given that not all biological systems are swarm-like (e.g., flower pollination algorithm [37], great salmon run [38], dolphin echolocation [39]). Other more generically natureinspired algorithms and frameworks mimic physical or chemical laws instead of biological ones. Notable examples of these type of algorithms include simulated annealing [40], spiral optimization [41], water cycle algorithm [42], and galaxy-based algorithm [43].

Given this plethora of different approaches and sources of inspiration, where the borders are partially very blurry, it might be difficult to identify categories or processes that uniquely can be tagged as the recognized sources of inspiration for SI or not. Thus, instead of using semantic definitions or definitions through specific inspiring animal systems, it may be better to characterize the source 
of inspiration of SI algorithms through collective properties and functionalities that can be designed after a system.

Given a collective system (i.e., a complex system featuring multiple interacting components, where the components are not necessarily living organisms), the key point to use it as a source of inspiration to design an SI system is to extract the why it works? and the how it works? Therefore, a first step towards an SI algorithm is the modeling of the system, which already leads to abstraction of the system down to its core functionalities. From there, further generalization can yield simple disembodied algorithms, or after translation from one physical domain to another, even totally differently embodied systems, e.g., in SI for CPSs. Good sources for this procedure of extracting system-level phenomena of interest and distilling them into algorithmic or embodied functionalities are all complex systems that show one of the following core collective capabilities:

- Collective decision making: This capability describes the behavior of systems that can collectively choose one option over one or several other options, e.g., ants choose shortest paths using pheromones, bees decide on the best foraging area, or on the best nest location $[23,44,45,26]$. Within such systems we find mostly positive feedback loops - the local behavior is reinforced and activates other elements of the system to participate governing the global (macroscopic) behavior of the system population. The challenge is to identify the microscopic proximate mechanisms that produce these collective phenomena.

- Collective regulation and homeostasis: Another interesting feature of complex systems, and mostly of biological organisms, is collective regulation aimed to keep specific system variables around a viable and efficient set point $[46,47]$. We often find such collective mechanisms in resource allocation mechanisms of social animals, e.g., collective food processing in ant [48] and bee colonies [49], regulation of building material in paper wasp nests [50], and collective brood care in honeybee hives [51]. In such systems, the crucial point is to identify the regulatory negative feedback loops 

rithms define themselves less often by their source of inspiration, but more by the way they perform their operations. More specifically, inspiration for SI is not restricted to natural systems, but rather to any complex system featuring an ensemble of interacting components that can display useful properties such as collective decision-making, regulation, homeostasis, and periodic patterns.

\subsection{Swarm models of Cyber-Physical systems}

As pointed out in the Introduction, a CPS encompasses different aspects of optimization, networking, and physical embodiment: it precisely merges and interleaves software agents, mechatronic devices, and communications [1, 3]. We nisms that ultimately produce the macroscopically observable homeostasis on the population level are to be identified.

- Collective periodic patterns: Collective systems can also show complex dynamics of state changes over time, sometimes even producing interesting complex spatial patterns like circular or spiral waves [52]. Due to state changes, such spatio-temporal waves can be exploited for collective computation, self-assessment of the swarm states (e.g., estimating the local group size), or signaling purposes $[53,54]$. The characteristic oscillations in such systems originate either in communication delays or in complex internal state-changing mechanisms of the involved agents. The oscillations in the end affect the robustness and the time constants of the aforementioned feedback loops that govern the macroscopic system behavior. Coupledoscillator systems and excitable media are among the most prominent examples of such natural systems.

To summarize the discussions of this section, we can say that today SI algocan model a CPS as a swarm of multiple components, where each component

that allows control. Also here the involved microscopic proximate mecha-

- integrates one or more physical devices (sensors, actuators, communication, memory, processor, etc.), 
- acts autonomously (i.e., control is distributed and/or decentralized),

- responds and possibly adapts to changing conditions, and

- locally communicates and interacts with other swarm components to possibly produce effective and useful behaviors at the system level.

For successfully developing SI solutions for swarms of CPSs it is important to consider both the physical and cyber aspects of CPSs. Furthermore, it is important to consider not only a single CPS but also ensembles of CPSs forming a CPSoS as defined in the Introduction that makes swarms of swarms operating at different spatial and temporal scales under different integration constraints.

For instance, we can describe an autonomous car with all its components as a CPS. At a smaller scale, each of the major components of the car (e.g., the Antilock Braking System (ABS)) can be described as CPSs. At a larger scale, a fleet of autonomous cars interacting and communicating with each other represents a swarm of CPSs. In order to acquire real-time sensor and traffic data, the cars in the swarm would also interact with further CPSs that are part of the surrounding environment and the supporting infrastructures. These CPSs form yet another swarm enabling interaction between different swarms of swarms. As there is no general limit to the number of devices and number of swarms, we can naturally arrive at a large-scale, multi-leveled swarm system integrating many interacting CPSs.

This simple yet realistic example shows that we expect the scale of the CPS swarms that we will have to deal with in the near future is extremely large. Moreover, these swarms will feature a high diversity on many scales. First, the swarms can have structural differences as well as differences on the individual level, both in CPS hardware and software. Second, the spatial scale ranges from close proximity interaction, such as two autonomous cars that interact in order to avoid collisions, to large-scale interactions between traffic guidance systems that must interact over parts of the city to optimize flows. Third, different CPSs work on different temporal scales, e.g., real-time reactions for emergency breaking or traffic profiling for traffic flow optimization. 
In order to map SI solutions and frameworks to this diversity and complexity of CPSs, it is convenient to make first a taxonomic effort in order to categorize the most relevant aspects of CPSs. We start it by noting that individual CPS properties can be roughly divided into capabilities and constraints.

A CPS can have very diverse capabilities. We identified the most important classes of capabilities to be mobility, communication, sensing, actuation, and processing [55]. For instance, mobile robots and autonomous cars have a high degree of mobility, while infrastructure-based CPSs such as used in smart grids have no or only little mobility. Communication capabilities are crucial when we talk about swarms of networked CPSs. Some CPSs such as sensor networks in IoT scenarios might have constrained wireless connections whereas others such as those used in industrial process control might have high capacity network access. For interacting with the physical world, it is crucial for CPSs to have sensing and/or actuation capabilities. In many cases it might be enough to have sensors to monitor the environment. Other applications such as smart homes require the CPSs to manipulate the environment, e.g., by closing the blinds or dimming the light. Finally, the processing capabilities allow the CPSs to process data and make informed decisions. Depending on the processing power of individual CPSs, some tasks might be delegated between devices to enable an efficient use of the processing resources.

On the constraints side we have limited resources in time and space [56]. Time constraints include limited energy supply which allows the CPSs to work only for a limited time period, limited processing power that defines the minimum required time for a given computation, and limitations on communication, sensing, and actuation that constrain the rate at which CPSs can perceive or manipulate their environment. Spatial constraints include kinematic constraints such as limited maneuverability, limited communication range, and the physical dimensions of individual CPSs that can inhibit them to enter confined spaces.

For the CPS swarm properties, the CPSoS scenarios, we look at the features that the CPSs exhibit as an ensemble. These features can be split into those related to swarm composition and those about the network topology that allows 
communication among the individual CPSs in the swarm. The swarm composition parameters include the size of the swarm, the spatial re-configurability which is closely related to the mobility and communication capabilities of individual CPSs, and the diversity of the CPSs that constitute the swarm $[57,58]$. The network topology is mainly defined by the communication capabilities of individual CPSs. Nevertheless, when forming a network of connected CPSs, different topologies can be chosen for the swarm [59]. For example, mesh networks are typically a good choice since they allow all swarm members in communication range of each other to exchange messages. In some cases it might make sense though to have a hierarchy and only exchange messages with higher layers of the hierarchy. One example are sensor networks, where the information sensed by individual CPSs needs to be forwarded to a central CPS in charge of consolidating, processing, and forwarding the data.

\section{Challenges using Swarm Intelligence}

While the adoption of SI approaches in CPSs can potentially bring potential advantages in terms of adaptability, robustness, and scalability of the systems 485 (see Section 2.1), it is also true that the use of a SI design comes with a number of challenges and drawbacks. In the following subsections we discuss the main limitations and challenges that can be associated to the use of SI design in general, and in the context of CPS swarms specifically. At the end of the section we provide suggestions about how to overcome or deal with these issues.

\subsection{Challenges deriving Formal Properties}

When designing a system, fundamental questions regard the capability to make predictions about the behaviors of the system. In turn, predictability is related to controllability that affects the ability to let the system achieving desired configurations. Predictability and controllability are properties that are inherently hard to achieve in complex systems design following a bottom-up approach, such as SI. 
A number of works have addressed these topics, a few recent developments are related to network science [60] and to the observability itself of complex systems [61]. Moreover, systems are usually described as dependent on a set of parameters (numeric and structural) whose effects on the resulting dynamics can be somehow extreme in a complex system, resulting in phase transitions $[62,63$, 64], self-organized criticality [65], and in deterministic chaotic behaviors [66, 67]. This is to say that in general, not only predictability and controllability are challenges, but also any parameter setting can have a major impact on the resulting dynamics of a SI system.

In the specific case of swarm systems that are also CPSs, the situation is even more challenging. CPSs are in fact "characterized by the tight interaction between a digital computing component (the cyber part) and a continuous-time dynamical system (the physical part)" [68]. This amounts to the coupling between two complex systems that makes it extremely hard to reason for instance in terms of model checking for deriving formal properties. Moreover, CPSs are plugged in the real-world, making them exposed to external random disturbances that are hard to model, or would require adaptive runtime models to be understood. The difficulty lies in creating models that are precise enough for timely adaption in order to exhibit the desired resilience to the dynamicity of the environment [69].

Coupling the inherent difficulties in obtaining formal guarantees from both sides, SI and CPSs, impose challenges and restrictions on the design and use of CPS swarm systems. This also says that SI systems may find limited use in life-critical applications such as the control of an airplane, precisely because of the lack of a clear definition of the possible behaviors of the system.

In SI literature, a quite extensive body of work has tackled the analysis of formal models in the context of disembodied SI (e.g., addressing optimization and decision-making scenarios), while embodied SI (e.g., swarm robotics and general CPSs) fall shorter in this respect because of presenting additional challenges.

In SI approaches for disembodied scenarios, mostly in optimization problems 
(e.g., ACO and PSO), the majority of the efforts towards formal properties are about stability and convergence. However, how it is discussed in [70] (for PSO, but similar considerations hold for ACO), analysis is often performed on simplified or restricted models mainly by removing algorithmic components or by imposing specific assumptions (e.g., for the PSO, using a random dynamical systems [71], applying Lyapunov stability analysis [72], and deriving graphical parameters from dynamic system theory [73]). Furthermore, it is often restricted to asymptotic properties of convergence per se, rather than dealing with the more difficult (and interesting) aspects of speed of convergence [74]. In general, in a number of practically used variants, PSO and ACO models rapidly become too complex to be amenable to theoretical analysis. Often, the only way for a performance analysis or parameter tuning is the use of empirical procedures possibly restricted on the assumption of defined theoretical properties (e.g., for the PSO by using a specifically designed objective function for convergence analysis [75], reviewing self-adaptive PSO algorithms related to stable points in parameter selection [76], studying continuous optimization algorithms for parameter tuning for swarm algorithms in general [77], applying experimental analysis of how particular design choices affect the quality and the shape of the Pareto front approximations generated in multi-objective ACO [78]).

In the case of embodied SI, giving formal guarantees for predictability and controllability seems to be more problematic compared to the above case of disembodied SI. Each real-world problem scenario presents its own peculiarities, that result in different performance objectives, metrics, constraints, requirements, and disturbances. At the same time, the physical characteristics and the behaviors of the individual CPSs composing the swarm (e.g., the robots) are different from implementation to implementation. Putting the two things together, the result is that each scenario, together with the specific choice of individual CPSs that apply specific behaviors, sets a new problem in practice. In this context it becomes difficult to abstract properties and reuse results across different works. However, by some mathematical abstraction, it is still possible to identify and perform an analysis of typical classes of swarm behaviors that include, 
among others, foraging [79, 80, 81, 82], coverage [83, 84, 85, 86], aggregation and pattern formation [87, 88, 89, 80], and cooperative tracking [83, 90, 91] (an extensive, and well discussed list of swarm behaviors can be found in [92, 93], and an extension in [13]).

\subsection{Challenges Designing the Local Rules}

SI design follows a bottom-up approach, where the global behavior is implicitly specified through the definition of local interaction rules: a system designer has to solve an inverse problem.

In the case of disembodied SI scenarios, such as optimization, it is relatively easy to heuristically define the rules that can lead to a desired global behavior. Instead, in the case of CPS swarms this is considerably more complex due to the multi-faceted, uncertain, and hardly predictable structure of the environment. Most of the work in this direction has addressed the case of the automatic synthesis of behavioral and interaction rules in swarm robotic systems.

In [94] artificial evolution techniques (provided by the FREVO [95] software) are used to generate neural controllers for homogeneous robot soccer teams; [96] applies novelty search to generate neural controllers for homogeneous robot swarms; in [97] the authors generate robot controllers by combining the use of an evolutionary approach with a formal language; in [98] supervisory control theory, a formal language based on a discrete-event representation of the system is used to automatically generate controllers that come with built-in proofs and have wider reusability across different robot systems; instead, in [99] the automatic design of control software for robot swarms is obtained by the generation of a probabilistic finite state machine resulting from an optimization process that maximizes a task-specific objective function; in [100] different evolutionary approaches are used for the generation of task-allocation mechanisms.

All these works (among others) provide valuable contribution to the automatic generation of rules that can be reused or expanded for the more general case of CPSs. Nevertheless, it must be acknowledged that they have been validated only in relatively simple scenarios, and have been applied only for 
homogeneous swarms. Therefore, these methods can hardly account for the

heterogeneous swarms of CPSs. Moreover, they incur into a dimensionality curse once the search space becomes reasonably large and complex.

Therefore, as CPS swarms require the synthesis of complex behaviors, current automatic generation systems might be unfeasible to use, at least in a systematic way. It can be noted that in any case, a convergence between modelchecking methods [101] in general CPSs, and automatic rule synthesis and verification in physical swarms will be needed to provide some guarantees in both design and control stages of a CPS swarm.

\subsection{Real-World Deployment Challenges}

So far, the large majority of the works that have been deployed and tested on physical swarms are in the domain of swarm robotics. Furthermore, they have been restricted to the use of small, simple and rather minimalistic robots, equipped with simple sensors, exchanging minimal amounts of data (e.g., in [102, $103,104])$. As a result, and in spite of the interesting self-organizing behaviors that might be observed, most of these works are proof-of-concepts that can hardly scale to useful applications in the real world: hardly they could be ported to complex real-world tasks in a reliable, predictable, and efficient manner.

Instead, nowadays impressive individual CPSs and robotic systems are being built, such as self-driving cars (Uber, Google), incredibly stable quadruped robots (Spot from Boston Dynamics), and advanced humanoid robots (Atlas by Boston Dynamics, Sophia by Hanson Robotics, Asimo by Honda). All these examples are extremely complex, from a mechatronic, algorithmic, and behavioral point of view. Compared to them, the tasks performed by currently implemented robot swarms look much alike toy examples. The question is whether swarm robotics, or rather CPS swarms, will be able soon to pass from the stage of studying group behaviors in quite abstract terms to the stage of producing systems that can reliably do useful things in the real world. This might require to pass from current oversimplified robot models and controls, to models and controls that are based on a more convenient trade-off between simplicity of 
design and capability of doing complex things in an effective and reliable way.

This might include to pass from an extremely parsimonious use of resources and information to a more intensive use of sensor data and information sharing.

The example of the Intel's swarm of Shooting Star drones [105] is exemplary in this respect. For the 2018 Winter Olympics opening ceremony, an impressive air show with 1,218 drones was performed by Intel (previous air shows were performed around the world with less drones). The system is labeled as a swarm and it is in terms of numbers, but it is not a swarm-intelligent system: it is actually a partially distributed system with a central controller. Individual drones get pre-computed trajectory scripts, and rely on a very precise external positioning system to find and adjust their way. The take-home message is: in order to do things in the real world, it might be necessary to give up some overly restrictive assumptions, and be pragmatic and balancing when integrating SI bottom-up design with top-down methods whenever possible.

A further challenge that is aligned with deployment of real systems is the maintenance of a deployed swarm system. Analog to the discussion in [106], despite being a swarm system, a complex technical system that must operate over a considerable lifetime will require maintenance in order to continuously provide its service during an extended system lifetime. It is an open question if maintenance of a technical system with such properties will be easier or more complicated to maintain than a traditionally designed technical application. In principle maintaining a system with many autonomous components is expected to be a difficult task. Indeed, the task might becomes easier if the same presence of multiple components is used to monitor and maybe fix problems from within the system. An example is provided in [107], where failed robots are detected and isolated in a timely manner from within the robot swarm.

\subsection{How to Address the Challenges}

We have already discussed a few general limitations of SI. In order to better support formal analysis and a systematic reuse of results, a possible way is more solidly framing SI in the context of the fields of complex systems and network 
science. This framing will ease the adoption of the sophisticated mathematical and modeling tools that are extensively used in the study of complex systems for characterizing time-evolution and stability properties, as well as analyzing the systems in terms of their structural and topological properties. In particular this latter has gained considerable attention in recent times, since information flows are keys to prime self-organization and emergence [108, 109].

Moreover, in the same line of developing the connections with related fields, the relationship between SI and the fields of game theory and multi-agent systems [110] should be acknowledged. Hence, this fact has not been exploited adequately, which can be beneficial to the study of the formal properties of SI systems. Game theory's equilibrium concepts and designing of interaction rules by adopting (and possibly adapting) mechanism design approaches [111, 112] could be a valid, sound alternative to current approaches for automatic rule generation.

When considering the limited applicability of current CPS swarms, we foresee that the study of physical swarms should move closer to the field of multiagent systems, while keeping an emphasis on self-organized behaviors and scalability. Actually, the difference between swarms and multi-agent decentralized systems is not sharply defined. For instance, from a recent survey on multirobot systems [113], the properties that are invoked for an algorithm of distributed control are locality of sensing and communications, scalability, safety, emergence, and the tasks that are considered are very much aligned with those in [93] considering swarm robotics. This says that differences are blurred, and sometimes it is just a matter of terms rather than substance.

\section{Future Trends and Directions}

As pointed out in the introduction, the world is featuring the increasing presence of complex dynamic systems that are increasingly collective and connected. These systems are cyber or physical, or, more generally both, cyber-physical. Given the nature and characteristics of the systems, we envisage that SI will 
play a major role addressing forthcoming challenges. Therefore, novel swarm models might be needed and novel application fields will open up. In the following subsections we discuss from where novel inspirational models could come from, in which kind of future applications SI will prove to be a prominent tool, and what the general open research topics in swarms of CPSs are.

\subsection{Future Inspirations}

SI models and algorithms have been mainly inspired from the observation of social interactions taken from models that were originally made on animal groups and societies [114]. If we compress these models down to the main functional building blocks required in most of SI models, we can identify the essential microscopic and local interaction rules that are effective in many agent systems by producing self-organized behavior that can emerge at macroscopic system level. In this perspective, we can think of enlarging the spectrum of potential inspirational models to include organic, inorganic, and social systems that show similar core properties. The goal is to build and extract abstract models from all natural sources of inspiration in a way that we explore time-effective, decentralized techniques from the specific scenarios studied in various research disciplines, and employ them to build new models and tackle the complex problems of real-world CPS applications.

The following list discusses specific fields that can provide new ideas for developing SI models. In most of the cases, these fields have already been exploited for some SI development. Our claim is that their potential has not really been unleashed in this respect, such that they can provide (more) disruptive inspirational contributions.

\subsubsection{Biology}

Animal societies have been the main source of inspiration in SI. In most cases, these systems have been considered in terms of purely collaborative societies, while predator-prey systems, and more in general, conflicting scenarios have attracted much less attention from SI practitioners [114]. However, to deal with 
cyber threats, as well as in the perspective of the interaction among multiple and possibly conflicting or competing CPSs, more attention should be devoted to non-collaborative systems. In this general field, a vast amount of research has been performed in the context of population dynamics [115], game theory [110], and evolutionary game theory [116]. We expect that ideas and models from these scenarios and research fields could be fruitfully integrated in the design of novel SI systems. Integration of autonomous robots into existing animal societies $[117,118,119]$ has been performed several times successfully with honeybees [120, 121], fish [122, 123, 124], cockroaches [125] and cows [126]. This is an emerging field of science, as embedding robots in living animal societies will allow both sides to bring in their special capabilities and to merge them in a symbiotic way, in order to generate a novel bio-hybrid system, a social cyborg.

(Programmable) Bacteria already serve as inspiration today. Their foraging [114] as well as their collective decision making process allows them to thrive and spread in difficult environments [127]. Furthermore, bacteria are programmable by altering their genetic code [128]. These characteristics could allow a swarm of bacteria to deliver drugs to tumors inter organic [129].

\subsubsection{Chemistry and Physics}

Molecular networks are studied in different disciplines [130], but especially for complex diseases. Networks are used to model molecular interactions to let the complex structure of, e.g., cancer or schizophrenia, being understood [131]. Therein, the nodes represent molecules (e.g., genes, RNA or proteins) and the edges represent relationships between them. Such network structures cân give inspiration for designing the complex interactions between agents in a swarm. An outstanding property of molecular-level interaction networks is their scalability and resilience. The physiological interaction network of a single cell comprises many thousands of chemical reactions altering the concentration levels of hundreds or thousands of chemical components. Even though these networks are extremely complex and of enormous size, their dynamics are still reacting quickly and flexible by staying, in parallel, within precisely controlled bounds, 
otherwise these organisms would quickly die. These properties of flexibility, robustness, scalability and emergent complexity arising from simple interactions have striking similarities with the desired features of SI. The properties of molecular networks, as well of the above-mentioned bacterial interaction patterns, can be studied in laboratory experiments under controlled conditions, as each study site is just a few microliters of fluid in a cuvette or a small agar plate on the lab desk. This makes bacterial and molecular networks excellent candidates for systematic searches for sources of inspiration of future SI algorithms.

Nanonetworks are interconnected nanomachines (arranged set of molecules) that have several abilities including computation, data storage, sensing, and actuation. Most of the nanonetwork-research is related to their non traditional communication types: electromagnetic or molecular communication [132]. SI could get inspired especially by the molecular communication between cells using synthesis, transformation, emission, propagation, and reception of molecules $[133]$.

\subsubsection{Human Cognition}

Also humans use certain strategies for interacting with each other to solve a task [134, 135, 136], e.g., collective patterns in crowd dynamics. To find the corresponding behavioral rules, experimental studies are applied, e.g., for single avoidance tasks among pedestrians [137]. For example, in [138] they observed environmental distributed humans with limited perception to solve a collective coordination task. This was one of the first experiments, before designing formal strategies to learn human-inspired behavior and maybe adapting it to CPS swarms as well. Overall, compared to the plethora of algorithms derived from (eu)social animals, it seems that almost no work was done trying to extract behaviors from human groups or societies in order to convert them into SI algorithms [139]. This is in fact very surprising, because humans as sources of inspiration can be easily queried by natural language and, as humans form the most-advanced and most complex societies and cultures, thus one would assume that these are the richest source of inspiration for SI algorithms. 


\subsubsection{Interdisciplinary Approaches}

Active matter, much like a swarm, is composed of many individual agents that follow simple rules and give rise to an emerging behavior for collective mocreate new structures of similar kind. The authors in $[142,143]$, e.g., describe collective motion in active solids and active crystals that apply elasticity-based mechanisms to realize self-organization. These models can be applied to the collective motion of CPS swarms such as pattern formation or morphogenesis.

They are especially suited for CPSs as they exhibit robustness to heterogeneity that can be analytically derived.

\subsection{Most promising Future Applications}

While so far SI implementations in the real world are quite limited, we acknowledge the fact that SI ideas and methods can potentially find their application in a wide spectrum of practical scenarios. Already Bonabeau and Meyer stated that the application of SI is only limited by imagination [16]. This statement strongly supports our hypothesis, stated in the introduction, about the ubiquitous presence of SI to tackle the challenges of the increasingly connected world. Especially in form of many variations of the ACO [144] and PSO [17] algorithms, SI has been applied to complex optimization scenarios in the real world, where typically the swarm members encode individual solution candidates. In contrast, there have been only few applications of physically embodied swarm algorithms up to now-most engineering solutions follow more traditional top-down or centralized approaches.

The intuition is that SI will increasingly find their application in real-world scenarios, when there is not enough information to solve the problem in an effec- 
tive top-down manner, a centralized approach would be computationally infeasible, or when real-time constraints inherently limit the ability to find optimal solutions. These are often the cases in complex CPSs, or in scenarios without existing infrastructure, where the requirement is to provide an ad-hoc working solution without the possibility to (re)establish an infrastructure framework first (e.g., after a disaster scenario [145]). More in general, we foresee the high potential of swarm applications when venturing from well-defined environments, such as well planned factory plants or warehouses into more complex environments such as traffic systems, urban environments, social networks, extreme/hostile environments, or unexplored territory.

More specifically, we can ask what will the next frontier be for the application of SI in CPS swarms. Here we point to specific application scenarios that are (partially) not possible today but show the potential for fully exploiting swarm characteristics. This is also indicated by the many pieces of research works, that are sometimes (only) in need of a system integration to confront future application requirements. In the following examples we envision the application of CPS swarms, describe their swarm characteristics, and indicate a set of open points still asking for research work to reach the desired application. For reasons of readability, we use the term CPS swarm only which serves as umbrella topic for the multiple different technologies (see Section 1).

\subsubsection{Autonomous Driving and Smart Traffic}

Autonomous driving is a trend topic nowadays, and asks for several computational complex tasks in a dynamic environment with real-time processing capabilities. That is where the concept of SI plays a role: thousands of cars (using different types of levels of autonomy [146]) and road infrastructure cooperate to find a common solution. Several advantages arise in such a smart traffic management system if a swarm model for autonomous cars and infrastructure is applied, including a reduced highway load, coordinated building of an emergency lane, improved traffic throughput, and reduced carbon emission. One application could be the navigation in swarms or fleets including information 
from other swarm members, the infrastructure and the dynamic environment.

Modeling networked, autonomous cars and smart infrastructure as a swarm is both feasible and beneficial for several reasons as i) each CPS is able to retrieve and evaluate information locally and follows local rules; ii) the local information can be exchanged with other CPSs, and can form a global one or one at the swarm level; iii) the infrastructure is known in advance or faces slight dynamics, e.g., in the case of accidents or construction sites; iv) a central traffic management system would not be able to coordinate and manage each CPS taking part in the traffic, while the individual CPSs can locally cooperate possibly resulting in emerging behaviors.

While transport logistics is already a prominent application for the active usage of, e.g., ants [147, 148, 149, 150] and bees [151] algorithms pre-calculating and optimizing routes for dispatchers, the concept of smart traffic is rather untouched. Although, swarms of autonomous cars and smart infrastructure are very present in media $[152,153,154]$, setting up such swarms still requires multiple considerations especially in terms of swarm management: What is the joint motivation to form a swarm? What do CPSs need to have in common therefore (characteristics, properties, sensor data, etc.)? How to join or leave a swarm? What decisions should be taken to set preference parameterization, such as: Which decisions can favor the local driver goal over the swarm goal over a global smart traffic goal? What is the number of CPSs to form a swarm to draw a benefit? Integrating the data retrieved by the infrastructure to the swarm of autonomous cars could further enrich the decision process to reach the above mentioned goals. The technology to realize CPS swarms in smart traffic are partially already there: While local retrieval and evaluation of sensor data [155] is a standard in today's cars, there are already specific attempts for V2X [156] standardized communication protocols realized on manufacturer side, where V2X describes vehicle-to-vehicle (V2V) and vehicle-to-infrastructure (V2I) communication. V2V is the inter-car communication, V2I communication is realized between cars and smart infrastructure, which is the result of combining physical infrastructure (streets, cameras, traffic lights, etc.) with digital 
infrastructure (sensors, communication networks, etc.). Furthermore, communication technologies like 5G [157] or Ultra-Wideband [158] offer a high security level, low latency, and high data rates to allow self-localization and real-time data exchange among the CPSs. These achievements will enable a swarm of autonomous cars to exchange their local information and react on it accordingly in real-time on a dynamic, reactive way. For example, the authors in [159] describe a swarm behavior module step-wise from selecting and abstracting sensor data to path planning for an autonomous car. In their approach the swarm members are detected objects which were moving during observation. In [160] the authors develop local rules based on SI that enables the interconnected autonomous cars running efficiently on arterial roads. Furthermore, introducing mobility concepts is no longer restricted to research. The Hondas SAFE SWARM ${ }^{\mathrm{TM}}$ concept can improve traffic flow by taking information from vehicles ahead including onboard sensors and systems, and V2X communication [161]. For an efficient and effective implementation of a smart traffic system, we would probably need to start from the very beginning. This includes a whole new architecture for gathering, processing, and analyzing real-time data. Additionally to the swarm system, a central traffic management system (TMS) can be installed that can have several supportive roles. First, it acts as data submission system as local event information needs to be accessible for all other cars to plan their routes appropriately. Second, it should provide an overview of global traffic situations, with the capability to fuse individual swarm information on the road. The process of data exchange among and between cars and TMS is additionally enriched with information from smart road infrastructure.

\subsubsection{Emergency Response}

The availability of professional teams of first responders in emergency situations plays a critical role to save lives and/or restore primary services. Conventional teams of first responders include police, army troops, fire brigades, and rescue forces from civil protection agencies. Swarms of CPSs of several kinds (e.g., all-terrain or flying robots $[145,162]$ ) could support and facilitate emer- 
gency response activities by working in tight collaboration and cooperation with human teams, creating a heterogeneous, multi human-CPS swarm. Activities industrial or power plants, or environmental disasters (e.g., forest fires, floods, storms, earthquakes). CPS agents can possibly navigate through a destroyed or human-hostile environment more effectively and safely than humans, gathering multi-sensorial data, coordinating with humans to focus on spotted regions of interest (e.g., possible presence of survivors), remove obstacles, re-establish structures, and save lives. In more general terms, a CPS swarm can be used as a dynamic, distributed augmented sensor-actuator system that could be an invaluable tool to help human first responders to conduct missions efficiently and effectively.

Establishing a mixed team with swarms of CPSs is feasible as long as i) each individual swarm member is capable to act according to local rules and is independent of a fixed infrastructure; ii) the infrastructure is typically transformed, paths are blocked, others are rather unstable, thus forming a dynamic environment; ii) individual information can be exchanged with other members, other swarms or the local control stations; iii) some dynamic ad-hoc communication infrastructure is established to replace the pre-existing one, usually not (anymore) available, allowing for intra and inter swarm communication and communication from the swarm to the control centers; iv) high-level decision-making can be performed at the control centers if feasible, balancing local autonomy vs. ystem-level decision-making; v) swarm members and human teammates can locally interact and exchange information in a transparent way; vi) cooperative and collaborative behaviors are in place at individual level such that effective behaviors can emerge at global level.

The effective real-world deployment of a mixed team (heterogeneous swarm) requires still substantial progress especially in terms of the capability of autonomous decision-making, data exchange and fusion, and acting in complex, uncharted environments. In heterogeneous swarms, e.g., drones and ground rovers cooperate to deliver first aid kids, or guide the victim to the closest 
emergency exit. For example, in Saez-Pons et al. [163] a swarm of autonomous robots was developed for SAR applications. They can generate a formation and navigate while keeping this formation using so-called social potential fields. There existed several other projects in this field $[164,165,166]$, but unfortunately none of them has produced applicable results for real-world missions, yet. Designing and deploying a heterogeneous swarm for such missions comes with a number of open questions, including number, type, and initial location of robots [167]. Especially, within the interaction of human teammates, intuitive and transparent ways for multi-modal dialogue and data exchange between humans and CPSs have to be designed [168] in terms of interfaces and easy control [169, 170, 171]. For an emergency response application (e.g., post earthquake) we mostly have a destroyed infrastructure, e.g., the communication infrastructure. Therefore, we need new relaying concepts, so that the swarm of CPSs is able to create its own communication network. For example, in Hauert et al. [172] a swarm of autonomous Micro Air Vehicles (MAVs) is developed to deploy and manage an ad-hoc WiFi network [173]. Moreover, the (destroyed) environment asks for high coordination and collaboration activities of the swarm member. This can be done with exchanging different status updates or, if the bandwidth allows, full images, detected points of interest, etc. Compact image processing algorithms aligned with the local processing capabilities is needed.

\subsubsection{Environmental monitoring}

Monitoring our Earth's threatened ecosystems is another future application scenario of autonomous large-scale CPS swarms. These swarms can act as an active sensor network that re-configures itself based on the data it has previously collected and evaluated in the habitat. In this way, points of interest can be monitored with a finer granularity, e.g., by aggregating a higher density of CPSs or by achieving a higher measurement frequency at the specific location.

Supporting environmental monitoring with a swarm of CPSs is feasible as i) each individual swarm member acts according to local rules to facilitate autonomous behavior; ii) the individual information is exchanged among the CPSs 
to decide on the new behavior; iii) the natural environment to be monitored is usually not known and subjected to natural dynamics including unforeseeable events; iv) in environmental monitoring the ecosystem may not be disturbed from extra-natural intruders, thus the system asks for high autonomy; v) to reach the goals described above, the individual swarm members need to cooperate leading to an emergent behavior.

Swarms are well-suited for operation in unforeseen environments, capable to compensate loss or failure of swarm members. Thus, this technology is an ideal candidate for long-term autonomous operation [174] during unforeseeable events in unknown environments. Lightweight and efficient communication protocols between CPSs [175] can be suitable especially in environmental monitoring, as each communication may disturb the natural communication network of the ecosystem. The less the CPSs communicate or act, the more undisturbed the environments will be, and the more the real natural behavior and processes can be analyzed. Thus, a regime like act only when needed but then act smartly and gently might be a suitable principle for such applications. Additionally, this sounds not too far away from the behavioral optimization that natural organisms would employ. Another advantage of lightweight and efficient communication is energy saving: such swarms are typically deployed to monitor the ecosystem over months or even years. Thus, an intelligent energy consumption needs to be aligned to the duration of observation. Such a CPS swarm for environmental monitoring could consist of actively moving agents. Thus, it can be capable of performing an important special task at the end of the monitoring: self-removal of the swarm from the ecosystems without leaving harm for nature in a eco-friendly and sustainable way. There are several successful realworld research projects on environmental monitoring using real-robots such as subCULTron [176], CORATAM [177], and CoCoRo [178].

\subsubsection{Electric Energy Grids}

Electric energy grids are large interconnected complex systems, which make them a candidate for swarm-based solutions. Venayagamoorthy [179] identi- 
fies several application fields of computational intelligence in electrical energy grids, among them energy and power flow management algorithms, voltage and reactive power control, forecasting dynamic loads, and vehicle-to-grid (V2G). Although, swarm algorithms are not explicitly pointed out as example, many of these coordination tasks are potential applications of SI.

Besides applications of swarm-based optimization algorithms like PSO, there exist a number of approaches where a part of the electrical energy grid is directly modeled as the swarm. An example for coordinating houses in a neighborhood with a swarm-like approach is presented in [180] with a demand side management approach that uses geographically close coupling to ensure fairness between consumers of a neighborhood. In [181], Steber, Bazan and German describe a virtual mass storage system composed of small distributed battery energy storage units installed in households with a roof-top photovoltaic system. In [182], a concept named SmartGRID is presented, which provides a decentralized and interoperable grid scheduling framework using a SI approach. Ramachandran [183] proposes a hybrid swarm/immune-based auction system for coordinating generation and consumption in a smart microgrid. Another application of swarm-based energy grids is swarm electrification using microgrids [184]. Instead of a traditional top-down designed energy grid, swarm electrification builds upon the integration of small off-grid system into a connected microgrid. Such a swarm-based electrification is especially of interest for rural under-developed areas [185, 186].

Considering a swarm of CPS to model electric energy grids is of interest because i) there is an increasing number of renewable distributed energy resources (DERs) installed, the system becomes more and more distributed, ii) renewable energy plants like photovoltaic and wind power cannot adjust their output to compensate for higher demand, which makes central control difficult, and iii) with the electrification of transportation comes an increased power consumption caused by a high number of individual consumers. These issues motivate a transition from a centralized control of few, manageable power plants to a distributed swarm-like system where producers, storage, and consumers align 
themselves in a self-organized way.

The effective real-world deployment of CPS swarm models in electric energy grids still requires substantial progress in research. An open question is how systems can be validated and tested before deployment, since electric energy grids are critical infrastructure. Deploying new control strategies in even a small part of the system can become the source of oscillations in the system [187] and affect the overall system stability. This problem can be either addressed by simulation, where one has to mind the reality gap [188] or by applying CPS swarm models within smart microgrids [189] that are sufficiently large to benefit from a swarm-based approach while being encapsulated from the national grid.

\subsubsection{Space Missions}

Space missions are very attractive for swarms of CPSs. For example, swarms could be used to check for damage all around the hull of a communication satellite or to perform disposal of space debris in a coordinated way. Another example is a flock of glittering reflective spheres that could sweep past an asteroid in the solar system to measure the pull of its gravity. Exploration of water, raw materials, and even life on (exo)planets will be the first missions, before thinking of human colonization. Low-cost and fault-tolerance are key characteristics that could be fuililled by a swarm of a large number of CPSs when exploring unknown environments autonomously [190]. Furthermore, the data collected through the swarm should be returned to Earth stations. Therefore, e.g., the NASA Marsbees [191] should use the Mars rover as base and charging station. The Japan Aerospace Exploration Agency goes even further and envisions swarms of autonomous machinery to prepare the ground excavate, and build facilities for astronauts on the moon and Mars [192]. The first real-world tests in swarms of satellites, typically related to networking and communication in the different spheres, were and are already launched (Nodes [193], Proba-3 [194], KickSat [195]).

Using a swarm of CPSs for this type of visionary applications is feasible as i) each individual CPS uses local rules to facilitate autonomous behavior; ii) lo- 
cal information is exchanged among the CPSs and with a station on the Earth (probably through a relay station in the orbit of the planet); iii) in such application scenarios information on the infrastructure is not given, the environment is dynamic and unknown; iv) a central control could not be used, related to the time-delay between operating swarm and human control; v) to reach the goals described above, the individual swarm members need to cooperate.

The aforementioned missions would still require expensive ground control to meet their objectives, nowadays. The vision is to make satellite formations autonomous - human intervention is costly in terms of communication delay between space and Earth. The formations are strongly dependent on the movement of a spacecraft: gravity, solar radiation pressure and atmospheric drag on a spacecraft in orbit influence the movement and causes the deviations from movement we know on Earth [196]. Guidance, navigation and control system are effected by the movement model that needs to be developed. As soon as planets can be explored directly on their surface, autonomous swarms can be deployed, whereby several points are open related to their transportation to the planet, their launch at the planet, navigation, examination of the ground, and finally returning the retrieved data to Earth including influences like planet-specific temperature, ground conditions, or gravity.

\subsubsection{Medical Applications}

Applications of swarms in medicine show an increased interest to overcome complex problems, e.g., in cancer cure. In health care, nanoparticles are important as they can leak out of blood vessels and go to target sites. These particles are too small to be programmed. Nevertheless, they can be made ready for using them in swarms: interaction can be achieved using changes of their coating, charge or size. If a group of nanoparticles shows swarm behavior, they could potentially travel to the target cancer cells, contain a coating of medicine, be activated by external stimuli, and destroy targeted tissue effectively. Whereas other medications diffuse into tissues, a swarm of nanoparticles has more intelligence and thus could more effectively target diseased tissue [197, 198]. 
Using swarms of nanoparticles can be supported as: i) each individual nanoparticle acts according to local rules to facilitate autonomous behavior; ii) the local information is exchanged among the nanoparticles and probably with an out-of-the-body monitoring station; iii) in such scenarios there is no infrastructure at all; iv) the environment is the human or animal body, partially unknown and highly dynamic, and iv) to reach the goals described above, the individual nanoparticles need to cooperate leading to an emergent behavior.

In such a cancer cure application with nanoparticles several basic questions are still open when it comes to form a swarm. For example, how the swarm communication is achieved between individual swarm members, between swarm members and environment, and between swarm and the outer-body world. Furthermore, the question is how to implement typical swarm characteristics related to complexity, local intelligence, and local processing. The swarm system needs to be simple, re-configurable, inexpensive, scalable, and verifiable. From these elaborations, we could get answers how to perform even more complex tasks including optimization, computation, decision-making, construction, selfassembly, and collective motion [197, 198]. This application vision is still at the very beginning. Nevertheless, the question arises, whether other medical applications could be realized by injecting a swarm to the human body.

\subsubsection{Human networks}

Human organizations and social networks driven by Web $2.0^{4}$ are further examples of collective systems. In particular, the swarm nature in social networks is apparent: Large numbers of highly heterogeneous individuals interact and communicate locally based on some notion of social neighborhood and continually generate global behavioral patterns (e.g., online trends, popularization of certain products or ideas). On the one hand, a SI methodology can be ap-

\footnotetext{
${ }^{4}$ Definition Oxford Dictionary "Web 2.0": "The second stage of development of the Internet, characterized especially by the change from static web pages to dynamic or user-generated content and the growth of social media."
} 
plied to study human networks, using their huge amount of online data sources, and perform analytical prediction and evaluation via simulation to forecast and maybe control the behavior of these inherently complex systems. On the other hand, swarms could find their practical application in many different ways in these humans networks. We have identified two main classes of applications: as an integral part of the human network, and as a physical service that the human network can use, trade, and negotiate. Nowadays, we are already using many types of digital assistants in our daily lives (e.g., Alexa [199], Siri [200], Cortana [201]) embodied in our physical world. They are probably one of the most advanced examples of CPSs operating in the real-world: they interact with humans (text-, speech-based), are fully connected, often mobile, do a lot of computation for a precise interaction with humans, are proactive proposing or reminding, etc. With the continuous developments they are more and more autonomous and capable in terms of decision-making, adaptation, and life-long learning. In future, we can expect that we will be able to delegate a number of tasks to these digital aliases, that can do the job for us potentially $24 / 7$. Once we will get at this stage, a society of digital us will be acting in parallel to our physical society, searching information for us, establishing connections, trading, etc.

A CPS swarm approach is a model for managing these billion-sized systems as i) the agents of the swarm are autonomous, possibly driven by a relative simple set of behavioral rules reflecting personality traits and preferences of the human counterpart; ii) they interact locally, based on some dynamic notion of social neighborhood, but can also gather and share global information; iii) the environments where the agents live, the forthcoming Web 3.0, and the physical world, are both highly dynamic, hard to predict, inherently parallel and distributed; iii) the same raison-d'être of a digital assistant is to provide services based on information gathering, that in the presence of a swarm of digital assistants requires continual information exchange, search, negotiation, and trading among the swarm members; iv) each agent is inherently selfish, being the alias of a (selfish) human, but socially-aware patterns can globally emerge by setting 
the right interaction rules.

Several useful applications of the digital assistants can be realized as CPS swarms. If consented (and with adequate privacy protection measures), our digital assistants will continually exchange information among them, such that it will be possible to know what is a CPS's and human's physical location. This information could play a phenomenal role in the case of epidemics to track potential exposures and automatically implement containment measures. Also in the case of emergency escape, swarm member assistants could build on local, real-time information exchange to let a safe escape plan emerging in a crowd by guiding the motion of the individual human counterparts. Circulation of fake news are a serious issue in social networks [202]. A limit to effectively spot fake news can be related to the lack of collaboration among different web miners employed by different entities (e.g., in [203]), that sets limits to the amount of gathered information and performed inference. Using a collaborative CPS swarm, a large amount of information can be effectively gathered in parallel, shared, used for miners coordination and to jointly perform powerful analysis and inference. Another possible future application of CPS swarms of digital assistants can be envisioned in finding, negotiating, and trading other CPS swarms that can act directly in the physical world. The idea is that multiple levels of CPS swarms interacting with each other. Currently, sensing-as-a-service (SaaS) is a business approach which is growing in the IoT field [204], where users pay for data gathered by specific sensors. This can naturally be extended with the use of more general CPS swarms [205], once endowed with enough mobility and autonomy for sensing and actuation (including manipulation). Therefore, at one level, CPS swarms are made available, offering sensing and actuation services at different locations. At another level, digital swarm members find and negotiate use and cost of available CPS resources to perform tasks of interest (e.g., clean a building, irrigate a field, monitor lack water, transport people), exchange experiences and mistakes to learn or get strategies for task solving by collaborating with each other. This will amount to set up a complex society of CPS swarms interacting at multiple levels among them. The blockchain 
technology, which is rapidly affirming as a fully decentralized and distributed system for secure transactions, will be a fundamental component to let this view of multiple CPS swarms acting upon the real-world happening. In fact, it will be essential to have a trust system in place, in order to guarantee security and correctness of all type transactions among swarm members [204].

\subsection{Research Challenges}

As SI is still considered as a rather young topic, there are several important open research question. Domain specific topics were already described in the previous subsections related to the individual visionary applications. For other applications, not mentioned here, exist many other research questions. However, they are strongly application-specific. General topics in swarms are independent of the domain or application. Nevertheless, we want to describe them on the example of swarms of CPSs.

There are several requirements a swarm of CPSs needs to deal with. This includes the design, programming, and implementation of highly distributed and connected digital technologies that are embedded in a multitude of devices. Beside these processes, there is the challenge to design increasingly autonomous physical systems with various dynamics and satisfying multiple critical constraints. In addition, we need to address systems of systems with a high degree of autonomy ensuring adaptability, scalability, robustness, complexity management, security and safety, and providing trust to humans in the loop. To get an overview on the open research topics, the general topics are split into following categories. They only address several general topics as the underlying details would run out of the scope of this paper.

\subsubsection{Modeling}

Forming models from natural or other inspirational sources is a big topic in the community. As mentioned in Section 4.1 there are still a lot of new sources of inspiration to be considered. This research also includes applicationspecific parameter fine-tuning for existing algorithms. In addition, algorithmic 
topics include algorithm scalability, rate of control, rate of convergence, and the trade-off between exploitation and exploration [206]. Moreover, a combination of different inspirational sources could be achieved to meet today's high complexity problems with self-organization capabilities. The main idea would be to customize application-specific SI algorithms to an underlying problem definition.

\subsubsection{Design}

The more swarms of CPSs are spread over different application contexts and the more massive their adoption, the more challenging becomes the issue of effectively designing systems able to reach pre-defined goals flexibly, reliably and adapting to changing surrounding conditions. Broad challenges that shall be addressed for designing next generation CPSs [207] include i) integration of complex, heterogeneous large-scale systems, ii) interaction between human and systems, iii) dealing with uncertainty, iv) measuring and validating system performance, v) enriching the systems with learning capabilities, and vi) system design. From a fundamental point of view, individual theories allowing formal description of the different aspects of CPS design including physical, technical, organizational, and human-system interaction are available, at different degrees of maturity. However, such disciplines are not fully integrated in a common systems theory. In other words, while methodologies, representations and tools exist for addressing single aspects of CPS design, a support to the whole design life cycle is still an open challenge. Furthermore, a formal design methodology must contain some kind of process that revisits and iteratively refines the micro level behaviors in order to create a bridge between local and global level behaviors. Although Brambilla et al. [92] describes behavior-based design and automatic design methods for swarms of CPSs, there is no implemented toolchain yet that is able to map these design processes. One approach for such a toolchain is given with the CPSwarm workbench [208]. It has the vision of combining several tools to guide a CPS swarm designer through the whole life cycle from design over optimization and simulation to deployment. 


\subsubsection{Validation and Verification}

Verification and validation in swarms of CPSs include a broad range of systems and systems-of-systems. These systems may include hardware, software, information, processes, personnel, and facilities. Reliable methodologies to validate a swarm of CPS need to be explored and, where possible, detailed standardized key performance indicators together with standardized scenarios, testbeds, and benchmarks need to be defined to ensure the re-use of measurement methodologies. Results should be fed back in order to enable necessary modifications in the design specifications, model definitions and validation activities and the subsequent re-engineering and validation of the affected artifacts.

\subsubsection{Human-in-the-Loop}

Enabling human interactions with the swarm system [209, 210] can provide a number of advantages since the CPS can potentially exploit humans' cognitive and sensory-motor capabilities, and, vice versa, human agents can use the CPS in closed loop interaction as an augmented external sensor-actuator system. The design and implementation of such mixed initiative systems [211] brings a number of scientific and technological challenges, ranging from how the interaction is physically performed in both directions (from single CPSs or CPS swarms to humans and vice versa), to how (and with which frequency) the complex state of the (distributed) CPS is presented to the human, to how the information or the commands from the human are disseminated to the CPS swarm. A lot of research interest has focused on developing interfaces and modalities for bidirectional interaction and dialogue, considering for instance the use of different multi-modal interfaces $[168,212,213,214]$ for proximal interaction with the swarm. Once a direct interaction of human operators in the loop of a swarm system is allowed, new potential risks also arise. In particular, system security, safety, stability, and reliability of response might get negatively affected by the presence of the human (i.e., if he/she acts irresponsibly or in a malevolent way). These aspects haven't attracted much attention so far but will be of a major concern in the near future, in particular the aspects of security and privacy that 
need to be guaranteed across all levels.

\section{Conclusions}

While originated out of a biological context, the concept of SI has been treated more frequently in the domain of technical systems. In this paper we focus on CPSs that link the physical world with a cyber representation and thereby address the vertical studies of systems such as cybernetics, mechatronics, design, and many more. The complexity of CPS interactions asks for a framework that is able to cope with the arising emergent behaviors. Swarm-based applications will play an important role when there is not enough information to solve the problem in a centralized way, when there are time constraints which do not allow to find an analytical solution, and when the operation needs to be performed in a dynamically changing enyironment. With an increasing complexity in upcoming applications this will mean that SI will be applied to solve a significant part of ubiquitous complex problems.

This paper provides research inspirations, topics and ideas to bridge the gap between the SI and CPS community. We are convinced that the following issues will pave the way: i) Further investigation on finding and using inspiring examples from biology, physics, medicine, cognitive sciences and interdisciplinary research for research on SI, ii) elaborating visionary, but useful applications beyond the current ones, and iii) driving forward research on SI addressing especially modeling, design, validation / verification and human-in-the-loop concepts.

\section{Acknowledgment}

The research leading to these results has received funding from the European Union Horizon 2020 research and innovation program under grant agreement No. 731946, CPSwarm project. 


\section{References}

[1] E. A. Lee, Cyber physical systems: Design challenges, in: Proceedings of the 11th IEEE International Symposium on Object Oriented Real-Time Distributed Computing, 2008, pp. 363-369.

[2] N. S. Foundation, Cyber Physical Systems, https://www.nsf.gov/ funding/pgm_summ.jsp?pims_id=503286, [Online; accessed 15-March2018].

[3] E. A. Lee, S. A. Seshia, Introduction to embedded systems: A cyberphysical systems approach, MIT Press, 2016.

[4] J. Zeng, L. T. Yang, M. Lin, H. Ning, J. Ma, A survey: Cyber-physicalsocial systems and their system-level design methodology, Future Generation Computer Systems 105 (2) (2020) 1028-1042.

[5] C. B. Nielsen, P. G. Larsen, J. Fitzgerald, J. Woodcock, J. Peleska, Systems of systems engineering: Basic concepts, model-based techniques, and research directions, ACM Computing Surveys 48 (2).

[6] Y. Liu, Y. Peng, B. Wang, S. Yao, Z. Liu, Review on cyber-physical systems, IEEE/CAA Journal of Automatica Sinica 4 (1) (2017) 27-40.

[7] S. Engell, R. Paulen, M. A. Reniers, C. Sonntag, H. Thompson, Core research and innovation areas in cyber-physical systems of systems, in: Proceedings of the International Workshop on Design, Modeling, and Evaluation of Cyber Physical Systems, Springer, 2015, pp. 40-55.

[8] P. Venkata Krishna, V. Saritha, H. Sultana, Challenges, Opportunities, and Dimensions of Cyber-Physical Systems, IGI Global, 2015.

[9] B. R. Ferrer, W. M. Mohammed, J. L. Martnez Lastra, A. Villalonga, G. Beruvides, F. Castao, R. E. Haber, Towards the adoption of cyberphysical systems of systems paradigm in smart manufacturing environments, in: IEEE 16th International Conference on Industrial Informatics (INDIN), 2018, pp. 792-799. 
[10] I. G. Vargas, T. Gottardi, R. T. V. Braga, Approaches for integration in system of systems: A systematic review, in: IEEE/ACM 4th International Workshop on Software Engineering for Systems-of-Systems (SESoS), 2016, pp. $32-38$.

[11] M. Dorigo, M. Birattari, et al., Swarm intelligence, Scholarpedia 2 (9) (2007) 1462.

[12] H. Hamann, Swarm robotics: A formal approach, Springer, 2018.

[13] M. Schranz, M. Rappaport, M. Umlauft, W. Elmenreich, Swarm robotic behaviors and current applications, Frontiers in Robotics and AI.

[14] G. Beni, J. Wang, Swarm intelligence in cellular robotic systems, in: Proceedings of the NATO Advanced Workshop on Robots and Biological Systems, 1989 , pp. 703-712.

[15] S. Garnier, J. Gautrais, G. Theraulaz, The biological principles of swarm intelligence, Swarm Intelligence 1 (1) (2007) 3-31.

[16] E. Bonabeau, C. Meyer, Swarm intelligence: A whole new way to think about business, Harvard Business Review 79 (5) (2001) 106-115.

[17] J. Kennedy, R. C. Eberhart, Particle swarm optimization, in: Proceedings of the IEEE International Conference on Neural Networks, 1995, pp. 19421948.

[18] M. Dorigo, M. Birattari, T. Stützle, Ant colony optimization: A computational intelligence technique, IEEE Computational Intelligence Magazine 1 (4) (2006) 28-39.

[19] E. Şahin, Swarm robotics: From sources of inspiration to domains of application, in: Proceedings of the International Workshop on Swarm Robotics, 2004, pp. 10-20. 
[20] M. M. Millonas, Swarms, phase transitions, and collective intelligence, C.G. Langton Edition, Vol. XVII, Santa Fe Institute Studies in the Sciences of the Complexity, Addison-Wesley Reading, Massachussetts, 1994.

[21] S. Camazine, Self-organization in biological systems, Princeton University Press, 2003.

[22] C. Prehofer, C. Bettstetter, Self-organization in communication networks: Principles and design paradigms, IEEE Communications Magazine 43 (7) (2005) 78-85.

[23] M. Szopek, T. Schmickl, R. Thenius, G. Radspieler, K. Crailsheim, Dynamics of collective decision making of honeybees in complex temperature fields, PloS one 8 (10) (2013) e76250.

[24] G. Sempo, S. Canonge, C. Detrain, J.-L. Deneubourg, Complex dynamics based on a quorum: Decision-making process by cockroaches in a patchy environment, Ethology 115 (12) (2009) 1150-1161.

[25] S. Kernbach, R. Thenius, O. Kernbach, T. Schmickl, Re-embodiment of honeybee aggregation behavior in an artificial micro-robotic system, Adaptive Behavior 17 (3) (2009) 237-259.

[26] R. Beckers, J.-L. Deneubourg, S. Goss, Trails and u-turns in the selection of a path by the ant Lassius niger, Journal of Theoretical Biology 159 (1992) $397-415$.

[27] M. Bodi, R. Thenius, M. Szopek, T. Schmickl, K. Crailsheim, Interaction of robot swarms using the honeybee-inspired control algorithm beeclust, Mathematical and Computer Modelling of Dynamical Systems 18 (1) (2012) 87-100.

[28] N. J. Gunther, A simple capacity model of massively parallel transaction systems, in: Proceedings of the Computer Measurement Group Conference, 1993, pp. 1035-1035. 
[29] T. Schmickl, H. Hamann, Beeclust: A swarm algorithm derived from honeybees. derivation of the algorithm, analysis by mathematical models and implementation on a robot swarm, in: Y. Xiao (Ed.), Bio-inspired Computing and Networking, CRC Press (Taylor \& Francis Group, 2011, Ch. 5, pp. $95-137$.

[30] F. Arvin, A. E. Turgut, T. Krajník, S. Yue, Investigation of cue-based aggregation in static and dynamic environments with a mobile robot swarm, Adaptive Behavior 24 (2) (2016) 102-118.

[31] R. Mayet, J. Roberz, T. Schmickl, K. Crailsheim, Antbots: A feasible visual emulation of pheromone trails for swarm robots, in: Proceedings of the International Conference on Swarm Intelligence, Springer, 2010, pp. 84-94.

[32] T. Schmickl, R. Thenius, C. Möslinger, G. Radspieler, S. Kernbach, K. Crailsheim, Get in touch: Cooperative decision making based on robotto-robot collisions, Autonomous Agents and Multi-Agent Systems 18 (1) (2008) 133-155.

[33] I. F. Jr., X. Yang, I. Fister, J. Brest, D. Fister, A brief review of natureinspired algorithms for optimization, Elektortehniški Vestnik 80 (3) (2013) $1-7$.

[34] Y. Tan, Y. Zhu, Fireworks algorithm for optimization, in: Proceedings of the International Conference on Swarm Intelligence, Springer, 2010, pp. $355-364$

[35] H. Sayama, Morphologies of self-organizing swarms in 3d swarm chemistry, in: Proceedings of the 14th Annual Conference on Genetic and Evolutionary Computation, ACM, 2012, pp. 577-584.

[36] X. Z. Gao, V. Govindasamy, H. Xu, K. Wang, K. Zenger, Harmony search method: Theory and applications, Computational Intelligence and Neuroscience 258491. 
[37] X.-S. Yang, Flower pollination algorithm for global optimization., in: Proceedings of the International Conference on Unconventional Computing and Natural Computation, 2012, pp. 240-249.

[38] A. Mozaffari, A. Fathi, S. Behzadipour, The great salmon run: A novel bio-inspired algorithm for artificial system design and optimisation, International Journal of Bio-Inspired Computation 4 (5) (2012) 286-301.

[39] A. Kaveh, N. Farhoudi, A new optimization method: Dolphin echolocation, Advances in Engineering Software 59 (2013) 53-70.

[40] S. Kirkpatrick, C. D. Gelatt, M. P. Vecchi, Optimization by simulated annealing, in: Readings in Computer Vision, Elsevier, 1987, pp. 606-615.

[41] K. Tamura, K. Yasuda, Spiral dynamics inspired optimization, Journal of Advanced Computational Intelligence and Intelligent Informatics 15 (8) (2011) 1116-1122.

[42] H. Eskandar, A. Sadollah, A. Bahreininejad, M. Hamdi, Water cycle algorithm - a novel metaheuristic optimization method for solving constrained engineering optimization problems, Computers \& Structures 110 (2012) $151-166$.

[43] H. Shah-Hosseini, Principal components analysis by the galaxy-based search algorithm: A novel metaheuristic for continuous optimisation, International Journal of Computational Science and Engineering 6 (1-2) (2011) 132-140.

[44] T. D. Seeley, S. Camazine, J. Sneyd, Collective decision-making in honey bees: How colonies choose among nectar sources, Behavioral Ecology and Sociobiology 28 (4) (1991) 277-290.

[45] T. D. Seeley, P. K. Visscher, Choosing a home: How the scouts in a honey bee swarm perceive the completion of their group decision making, Behavioral Ecology and Sociobiology 54 (2003) 511-520. 
[46] M. Kleinhenz, B. Bujok, S. Fuchs, J. Tautz, Hot bees in empty broodnest cells: Heating from within, Journal of Experimental Biology 206 (23) (2003) 4217-4231.

[47] T. Schmickl, K. Crailsheim, Inner nest homeostasis in a changing environment with special emphasis on honey bee brood nursing and pollen supply, Apidologie 35 (3) (2004) 249-263.

[48] T. Schmickl, I. Karsai, Sting, carry and stock: How corpse availability can regulate de-centralized task allocation in a ponerine ant colony, PloS one 9 (12) (2014) e114611.

[49] T. Schmickl, I. Karsai, How regulation based on a common stomach leads to economic optimization of honeybee foraging, Journal of Theoretical Biology 389 (2016) 274-286.

[50] I. Karsai, T. Schmickl, Regulation of task partitioning by a common stomach: A model of nest construction in social wasps, Behavioral Ecology 22 (4) (2011) 819-830.

[51] T. Schmickl, K. Crailsheim, Cannibalism and early capping: Strategy of honeybee colonies in times of experimental pollen shortages, Journal of Comparative Physiology A 187 (7) (2001) 541-547.

[52] A. Garfinkel, The slime mold dictyostelium as a model of self-organization in social systems, in: Self-organizing Systems: The Emergence of Order, Springer, 1987, pp. 181-213.

[53] T. Sato, T. Kano, A. Ishiguro, On the applicability of the decentralized control mechanism extracted from the true slime mold: A robotic case study with a serpentine robot, Bioinspiration \& Biomimetics 6 (2) (2011) 026006 .

[54] T. Schmickl, K. Crailsheim, A navigation algorithm for swarm robotics inspired by slime mold aggregation, in: Proceedings of the International Workshop on Swarm Robotics, Springer, 2006, pp. 1-13. 
[55] S. K. Khaitan, J. D. McCalley, Design techniques and applications of cyberphysical systems: A survey, IEEE Systems Journal 9 (2) (2015) $350-365$.

[56] J. Shi, J. Wan, H. Yan, H. Suo, A survey of Cyber-Physical Systems, in: Proceedings of the IEEE International Conference on Wireless Communications and Signal Processing, 2011, pp. 1-6.

[57] F. Xia, X. Kong, Z. Xu, Cyber-physical control over wireless sensor and actuator networks with packet loss, in: Wireless Networking Based Control, Springer New York, 2011, pp. 85-102.

[58] E. Yanmaz, S. Yahyanejad, B. Rinner, H. Hellwagner, C. Bettstetter, Drone networks: Communications, coordination, and sensing, Ad Hoc Networks 68 (2018) 1-15.

[59] Y. Del Valle, G. K. Venayagamoorthy, S. Mohagheghi, J.-C. Hernandez, R. G. Harley, Particle swarm optimization: basic concepts, variants and applications in power systems, IEEE Transactions on Evolutionary Computation 12 (2) (2008) 171-195.

[60] L. R. J. Gates, Control of complex networks requires both structure and dynamics, Nature, Scientific Reports 6 (24456).

[61] Y.-Y. Liu, J.-J. Slotine, A.-L. Barabási, Observability of complex systems, Proceedings of the National Academy of Sciences 110 (7) (2013) 24602465 .

[62] R. V. Solé, S. C. Manrubia, B. Luque, J. Delgado, J. Bascompte, Phase transitions and complex systems: Simple, nonlinear models capture complex systems at the edge of chaos, Complexity 1 (4) (1996) 13-26.

[63] C. Dabrowski, Catastrophic event phenomena in communication networks: A survey, Computer Science Review 18 (2015) 10-45. 
[64] T. Bossomaier, L. Barnett, M. Harré, Information and phase transitions in socio-economic systems, Complex Adaptive Systems Modeling 1 (1) (2013) 9 .

[65] N. W. Watkins, G. Pruessner, S. C. Chapman, N. B. Crosby, H. J. Jensen, 25 years of self-organized criticality: Concepts and controversies, Space Science Reviews 198 (1) (2016) 3-44.

[66] S. Strogatz, Nonlinear dynamics and chaos: With applications to physics, biology, chemistry, and engineering, Studies in Nonlinearity, Avalon Publishing, 2014.

[67] X. Zang, S. Iqbal, Y. Zhu, X. Liu, J. Zhao, Applications of chaotic dynamics in robotics, International Journal of Advanced Robotic Systems $13(2)$.

[68] E. Clarke, P. Zuliani, Statistical model checking for cyber-physical systems, in: T. Bultan, P. Hsiung (Eds.), Proceedings of the International Symposium on Automated Technology for Verification and Analysis, Vol. 6996, 2011.

[69] A. Bennaceur, C. Ghezzi, K. Tei, T. Kehrer, D. Weyns, R. Calinescu, S. Dustdar, Z. Hu, S. Honiden, F. Ishikawa, Z. Jin, J. Kramer, M. Litoiu, M. Loreti, G. Moreno, H. Muller, L. Nenzi, B. Nuseibeh, L. Pasquale, W. Reisig, H. Schmidt, C. Tsigkanos, H. Zhao, Modelling and analysing resilient cyber-physical systems, in: Proceedings of the International Symposium on Software Engineering for Adaptive and Self-Managing Systems, IEEE, 2019, pp. 70-76.

[70] C. W. Cleghorn, Particle swarm optimization: Empirical and theoretical stability analysis, Ph.D. thesis, Department of Computer Science, University of Pretoria, South-Africa (2017).

[71] A. Erskine, T. Joyce, J. M. Herrmann, Stochastic stability of particle swarm optimisation, Swarm Intelligence 11 (3) (2017) 295-315. 
[72] V. Kadirkamanathan, K. Selvarajah, P. Fleming, Stability analysis of the particle dynamics in particle swarm optimizer, IEEE Transactions on Evolutionary Computation 10 (3).

[73] I. C. Trelea, The particle swarm optimization algorithm: Convergence analysis and parameter selection, Information Processing Letters 85 (6) (2003) 317-325.

[74] W. J. Gutjahr, Mathematical runtime analysis of ACO algorithms: Survey on an emerging issues, Swarm Intelligence 1 (2002) 59-79.

[75] C. W. Cleghorn, A. P. Engelbrecht, Particle swarm variants: Standardized convergence analysis, Swarm Intelligence 9 (2-3) (2015) 177-203.

[76] K. R. Harrison, A. P. Engelbrecht, B. M. Ombuki-Berman, Self-adaptive particle swarm optimization: A review and analysis of convergence, Swarm Intelligence 12 (3) (2018) 187-226.

[77] Z. Yuan, M. A. Montes de Oca, M. Birattari, T. Stützle, Continuous optimization algorithms for tuning real and integer parameters of swarm intelligence algorithms, Swarm Intelligence 6 (1) (2012) 49-75.

[78] M. López-Ibáñez, T. Stützle, An experimental analysis of design choices of multi-objective ant colony optimization algorithms, Swarm Intelligence 6 (3) (2012) 207-232.

[79] E. Castello, T. Yamamoto, F. D. Libera, W. Liu, A. F. T. Winfield, Y. Nakamura, H. Ishiguro, Adaptive foraging for simulated and real robotic swarms: The dynamical response threshold approach, Swarm Intelligence 10 (1) (2016) 1-31.

[80] V. Gazi, K. M. Passino, Stability analysis of social foraging swarms, IEEE Transactions on Systems, Man, and Cybernetics: Part B 34 (1) (2004) 539-557. 
[81] F. Ducatelle, G. A. Di Caro, A. Förster, M. Bonani, M. Dorigo, S. Magnenat, F. Mondada, R. O'Grady, C. Pinciroli, P. Rétornaz, V. Trianni, L. M. Gambardella, Cooperative navigation in robotic swarms, Swarm Intelligence 8 (1) (2014) 1-33.

[82] N. R. Hoff, A. Sagoff, R. J. Wood, R. Nagpal, Two foraging algorithms for robot swarms using only local communication, in: Proceedings of the IEEE International Conference on Robotics and Biomimetics, 2010, pp. $123-130$.

[83] F. Zhang, A. L. Bertozzi, K. Elamvazhuthi, S. Berman, Performance bounds on spatial coverage tasks by stochastic robotic swarms, IEEE Transactions on Automatic Control 63 (6) (2018) 1473-1488.

[84] A. M. Schroeder, M. Kumar, Design of decentralized chemotactic control law for area coverage using swarm of mobile robots, in: Proceedings of the American Control Conference, 2016, pp. 4317-4322.

[85] A. Mahadev, D. Krupke, S. P. Fekete, A. T. Becker, Mapping and coverage with a particle swarm controlled by uniform inputs, in: Proceedings of the IEEE/RSJ International Conference on Intelligent Robots and Systems, 2017, pp. $1097-1104$.

[86] N. Correll, A. Martinoli, Robust distributed coverage using a swarm of miniature robots, in: Proceedings of the IEEE International Conference on Robotics and Automation, 2007, pp. 379-384.

[87] A. Gasparri, A. Priolo, G. Ulivi, A swarm aggregation algorithm for multirobot systems based on local interaction, in: Proceedings of the IEEE International Conference on Control Applications, 2012, pp. 1497-1502.

[88] G. Antonelli, F. Arrichiello, S. Chiaverini, Flocking for multi-robot systems via the null-space-based behavioral control, Swarm Intelligence 4 (1) (2009) 1-37. 
[89] A. E. Turgut, H. Çelikkanat, F. Gökçe, E. Şahin, Self-organized flocking in mobile robot swarms, Swarm Intelligence 2 (2) (2008) 97-120.

[96] J. Gomes, P. Urbano, A. L. Christensen, Evolution of swarm robotics systems with novelty search, Swarm Intelligence 7 (2) (2013) 115-144.

[97] E. Ferrante, E. Duéñez Guzmán, A. E. Turgut, T. Wenseleers, Geswarm: Grammatical evolution for the automatic synthesis of collective behaviors in swarm robotics, in: Proceedings of the 15th Annual Conference on Genetic and Evolutionary Computation, 2013, pp. 17-24.

[98] Y. K. Lopes, S. M. Trenkwalder, A. B. Leal, T. J. Dodd, R. Groß, Supervisory control theory applied to swarm robotics, Swarm Intelligence 10 (1) (2016) 65-97. 
[99] G. Francesca, M. Brambilla, A. Brutschy, V. Trianni, M. Birattari, Automode: A novel approach to the automatic design of control software for robot swarms, Swarm Intelligence 8 (2) (2014) 89-112.

[100] E. Tuci, A. Rabérin, On the design of generalist strategies for swarms of simulated robots engaged in a task-allocation scenario, Swarm Intelligence 9 (4) (2015) 267-290.

[101] E. M. Clarke, T. A. Henzinger, H. Veith, R. Bloem (Eds.), Handbook of model checking, Springer, 2016.

[102] M. Rubenstein, C. Ahler, N. Hoff, A. Cabrera, R. Nagpal, Kilobot: A low cost robot with scalable operations designed for collective behaviors, Robotics and Autonomous Systems 62 (7) (2014) 966-975.

[103] M. Jdeed, S. Zhevzhyk, F. Steinkellner, W. Elmenreich, Spiderino - a lowcost robot for swarm research and educational purposes, in: Proceedings of the 13th Workshop on Intelligent Solutions in Embedded Systems, 2017, pp. 35-39.

[104] F. Arvin, J. Murray, C. Zhang, S. Yue, Colias: An autonomous micro robot for swarm robotic applications, International Journal of Advanced Robotic Systems 11 (113) (2014) 1-10.

[105] Intel Corporation, Intel drone light show breaks guinness world records title at olympic winter games pyeongchang, https://newsroom.intel. com/news-releases/intel-drone-light-show-breaks-guinnessworld-records-title-olympic-winter-games-pyeongchang-2018/, [Online; accessed 30-September-2019] (2018).

[106] W. Elmenreich, H. de Meer, Self-organizing networked systems for technical applications: A discussion on open issues, in: J. S. K.A. Hummel (Ed.), Proceedings of the 3rd International Workshop on Self-Organizing Systems, Springer, 2008, pp. 1-9. 
[107] A. L. Christensen, R. OGrady, M. Dorigo, From fireflies to fault-tolerant swarms of robots, Transactions on Evolutionary Computation 13 (4) (2009) 754-766.

[114] R. Parpinelli, H. Lopes, New inspirations in swarm intelligence: A survey, International Journal of Bio-Inspired Computation 3 (1) (2011) 1-16.

[115] M. Iannelli, A. Pugliese, An Introduction to Mathematical Population Dynamics: A long the trail of Volterra and Lotka, Springer, 2014.

[116] W. Sandholm, Population games and evolutionary dynamics, MIT Press, 1620

[117] F. Mondada, A. Martinoli, N. Correll, A. Gribovskiy, J. I. Halloy, R. Siegwart, J.-L. Deneubourg, A general methodology for the control of mixed 
natural-artificial societies, Handbook of Collective Robotics (2011) 399428.

[122] F. Bonnet, R. Mills, M. Szopek, S. Schönwetter-Fuchs, J. Halloy, S. Bogdan, L. Correia, F. Mondada, T. Schmickl, Robots mediating interactions between animals for interspecies collective behaviors, Science Robotics 4 (28) (2019) eaau7897.

[123] F. Bonnet, Y. Kato, J. Halloy, F. Mondada, Infiltrating the zebrafish swarm: Design, implementation and experimental tests of a miniature robotic fish lure for fish-robot interaction studies, Artificial Life and Robotics 21 (3) (2016) 239-246.

[124] T. Landgraf, D. Bierbach, H. Nguyen, N. Muggelberg, P. Romanczuk, J. Krause, Robofish: Increased acceptance of interactive robotic fish with 
[130] M. Kanehisa, S. Goto, M. Furumichi, M. Tanabe, M. Hirakawa, KEGG for representation and analysis of molecular networks involving diseases

and drugs, Nucleic Acids Research 38 (suppl_1) (2010) D355-D360.

[131] K. S. Grennan, C. Chen, E. S. Gershon, C. Liu, Molecular network anal-
ysis enhances understanding of the biology of mental disorders, Bioessays 1675

K. S. Grennan, C. Chen, E. S. Gershon, C. Liu, Molecular network anal-
ysis enhances understanding of the biology of mental disorders, Bioessays 36 (6) (2014) 606-616.

realistic eyes and natural motion patterns by live trinidadian guppies, Bioinspiration \& Biomimetics 11 (1) (2016) 015001.

[125] J. Halloy, G. Sempo, G. Caprari, C. Rivault, M. Asadpour, F. Tâche, I. Saïd, V. Durier, S. Canonge, J. M. Amé, C. Detrain, N. Correll, A. Martinoli, F. Mondada, R. Siegwart, J. L. Deneubourg, Social integration of robots into groups of cockroaches to control self-organized choices, Science 318 (5853) (2007) 1155-1158.

[126] N. Correll, M. Schwager, D. Rus, Social control of herd animals by integration of artificially controlled congeners, in: Proceedings of the International Conference on Simulation of Adaptive Behavior, Springer, 2008, pp. 437-446.

[127] A. Shklarsh, G. Ariel, E. Schneidman, E. Ben-Jacob, Smart swarms of bacteria-inspired agents with performance adaptable interactions, PLoS Computational Biology 7 (9) (2011) 1-11.

[128] M. Jinek, K. Chylinski, I. Fonfara, M. Hauer, J. A. Doudna, E. Charpentier, A programmable dual-RNA-guided DNA endonuclease in adaptive bacterial immunity, Science 337 (6096) (2012) 816-821.

[129] O. Felfoul, M. Mohammadi, S. Taherkhani, D. De Lanauze, Y. Z. Xu, D. Loghin, S. Essa, S. Jancik, D. Houle, M. Lafleur, et al., Magnetoaerotactic bacteria deliver drug-containing nanoliposomes to tumour hypoxic regions, Nature Nanotechnology 11 (11). 
[132] I. F. Akyildiz, J. M. Jornet, M. Pierobon, Nanonetworks: A new frontier in communications, Communications of the ACM 54 (11) (2011) 84-89.

[133] I. F. Akyildiz, M. Pierobon, S. Balasubramaniam, Y. Koucheryavy, The internet of bio-nano things, IEEE Communications Magazine 53 (3) (2015) $32-40$.

[134] J. Krause, G. D. Ruxton, S. Krause, Swarm intelligence in animals and humans, Trends in Ecology \& Evolution 25 (1) (2010) 28 - 34.

[135] S. Krause, R. James, J. J. Faria, G. D. Ruxton, J. Krause, Swarm intelligence in humans: Diversity can trump ability, Animal Behaviour 81 (5) (2011) $941-948$.

[136] J. J. Faria, J. R. Dyer, C. R. Tosh, J. Krause, Leadership and social information use in human crowds, Animal Behaviour 79 (4) (2010) 895 901.

[137] M. Moussaïd, D. Helbing, S. Garnier, A. Johansson, M. Combe, G. Theraulaz, Experimental study of the behavioural mechanisms underlying selforganization in human crowds, Proceedings of the Royal Society of London B: Biological Sciences 276 (1668) (2009) 2755-2762.

[138] A. Tavakoli, H. Nalbandian, N. Ayanian, Crowdsourced coordination through online games, in: Proceedings of the 11th ACM/IEEE International Conference on Human-Robot Interaction, 2016, pp. 527-528.

[139] R. S. Parpinelli, H. S. Lopes, New inspirations in swarm intelligence: A survey, International Journal of Bio-Inspired Computation 3 (1) (2011) $1-16$.

1700 [140] T. Vicsek, A. Czirók, E. Ben-Jacob, I. Cohen, O. Shochet, Novel type of phase transition in a system of self-driven particles, Physical Review Letters 75 (6) (1995) 1226-1229. 
[141] T. Schmickl, M. Stefanec, K. Crailsheim, How a life-like system emerges from a simple particle motion law, Scientific reports 6 (2016) 37969.

[142] E. Ferrante, A. E. Turgut, M. Dorigo, C. Huepe, Elasticity-driven collective motion in active solids and active crystals, arXiv preprint arXiv:1301.2620.

[143] E. Ferrante, A. E. Turgut, M. Dorigo, C. Huepe, Collective motion dynamics of active solids and active crystals, New Journal of Physics 15 (9) (2013) 095011.

[144] M. Dorigo, M. Birattari, Ant colony optimization, in: Encyclopedia of machine learning, Springer, 2011, pp. 36-39.

[145] K. Daniel, B. Dusza, A. Lewandowski, C. Wietfeld, Airshield: A systemof-systems muav remote sensing architecture for disaster response, in: Proceedings of the 3rd Annual IEEE Systems Conference, IEEE, 2009, pp. 196-200.

[146] National Highway Traffic Safety Administration. (NHTSA), Preliminary Statement of Policy Concerning Automated Vehicles, Tech. rep. (2013).

[147] R. Montemanni, L. M. Gambardella, A. E. Rizzoli, A. V. Donati, Ant colony system for a dynamic vehicle routing problem, Journal of Combinatorial Optimization 10 (4) (2005) 327-343.

[148] B. Yu, Z.-Z. Yang, B. Yao, An improved ant colony optimization for vehicle routing problem, European Journal of Operational Research 196 (1) (2009) 171-176.

[149] M. Reed, A. Yiannakou, R. Evering, An ant colony algorithm for the multi-compartment vehicle routing problem, Applied Soft Computing 15 (2014) 169-176.

[150] Y. Zhang, Y. Yuan, K. Lu, E-commerce information system data analytics by advanced aco for asymmetric capacitated vehicle delivery routing, Information Systems and e-Business Management (2019) 1-19. 
[151] S. Senge, H. F. Wedde, Bee-inpired road traffic control as an example of swarm intelligence in cyber-physical systems, in: Proceedings of the 38th Euromicro Conference on Software Engineering and Advanced Applications, IEEE, 2012, pp. 258-265.

[152] D. Furlonger, Swarm intelligence: From smart cars to smart traffic, https://www.businesslive.co.za/bd/life/motoring/2017-0814-swarm-intelligence-from-smart-cars-to-smart-traffic, [Online; accessed 27-September-2019] (2017).

[153] I. H. Security, Fish swarm model for energy-saving autonomous vehicle swarm, https://i-hls.com/archives/84280, [Online; accessed 30September-2019] (2018).

[154] J. Hu, P. Bhowmick, F. Arvin, A. Lanzon, B. Lennox, Cooperative control of heterogeneous connected vehicle platoons: An adaptive leader-following approach, IEEE Robotics and Automation Letters 5 (2) (2020) 977-984.

[155] S.-W. Kim, Z. J. Chong, B. Qin, X. Shen, Z. Cheng, W. Liu, M. H. Ang, Cooperative perception for autonomous vehicle control on the road: Motivation and experimental results, in: Proceedings of the IEEE/RSJ International Conference on Intelligent Robots and Systems, 2013, pp. $5059-5066$.

[156] S. Chen, J. Hu, Y. Shi, Y. Peng, J. Fang, R. Zhao, L. Zhao, Vehicle-toeverything (v2x) services supported by lte-based systems and 5g, IEEE Communications Standards Magazine 1 (2) (2017) 70-76.

[157] J. G. Andrews, S. Buzzi, W. Choi, S. V. Hanly, A. Lozano, A. C. Soong, J. C. Zhang, What will $5 \mathrm{~g}$ be?, IEEE Journal on Selected Areas in Communications 32 (6) (2014) 1065-1082.

[158] K. Guo, Z. Qiu, C. Miao, A. H. Zaini, C.-L. Chen, W. Meng, L. Xie, Ultra-wideband-based localization for quadcopter navigation, Unmanned Systems 4 (01) (2016) 23-34. 
[159] F. Ulbrich, S. S. Rotter, R. Rojas, Adapting to the traffic swarm: Swarm behaviour for autonomous cars, in: Y. Tan (Ed.), Handbook of Research on Design, Control, and Modeling of Swarm Robotics, IGI Global, 2016, Ch. 10, pp. 263-285.

[160] L. Li, R. Hao, W. Ma, X. Qi, C. Diao, Swarm intelligence based algorithm for management of autonomous vehicles on arterials, Tech. rep., SAE (2018).

[161] H. Motor, Safe swarm, https://global.honda/innovation/CES/2019/ safe_swarm.html, [Online; accessed 01-October-2019] (2019).

[162] J. Scherer, S. Yahyanejad, S. Hayat, E. Yanmaz, T. Andre, A. Khan, V. Vukadinovic, C. Bettstetter, H. Hellwagner, B. Rinner, An autonomous multi-uav system for search and rescue, in: Proceedings of the First Workshop on Micro Aerial Vehicle Networks, Systems, and Applications for Civilian Use, 2015, pp. 33-38.

[163] J. SaezPons, L. Alboul, J. Penders, L. Nomdedeu, Multirobot team formation control in the GUARDIANS project, Industrial Robot: An International Journal 37 (4) (2010) 372-383.

[164] G. De Cubber, D. Doroftei, D. Serrano, K. Chintamani, R. Sabino, S. Ourevitch, The EU-ICARUS project: Developing assistive robotic tools for search and rescue operations, in: Proceedings of the IEEE International Symposium on Safety, Security, and Rescue Robotics, 2013, pp. 1-4.

[165] L. Marconi, C. Melchiorri, M. Beetz, D. Pangercic, R. Siegwart, S. Leutenegger, R. Carloni, S. Stramigioli, H. Bruyninckx, P. Doherty, et al., The SHERPA project: Smart collaboration between humans and ground-aerial robots for improving rescuing activities in alpine environments, in: Proceedings of the IEEE International Symposium on Safety, Security, and Rescue Robotics, 2012, pp. 1-4. 
[166] Project SWARMIX: Synergistic interactions in swarms of heterogeneous agents (2011-2014), http://www.swarmix.org/, [Online; accessed 14August-2019].

[172] S. Hauert, J.-C. Zufferey, D. Floreano, Evolved swarming without positioning information: An application in aerial communication relay, $\mathrm{Au}-$ tonomous Robots 26 (1) (2009) 21-32. 
[173] M. Varga, M. Basiri, G. Heitz, D. Floreano, Distributed formation control of fixed wing micro aerial vehicles for area coverage, in: Proceedings of the IEEE/RSJ International Conference on Intelligent Robots and Systems, 2015, pp. 669-674.

[174] E. Donati, G. J. van Vuuren, K. Tanaka, D. Romano, T. Schmickl, C. Stefanini, amussels: Diving and anchoring in a new bio-inspired underactuated robot class for long-term environmental exploration and monitoring, in: Proceedings of the Conference Towards Autonomous Robotic Systems, Springer, 2017, pp. 300-314.

[175] T. Schmickl, F. Wotawa, R. Thenius, J. C. Varughese, Fstaxis algorithm: Bio-inspired emergent gradient taxis, in: Proceedings of the Artificial Life Conference 2016 13, MIT Press, 2016, pp. 330-337.

[176] R. Thenius, D. Moser, J. C. Varughese, S. Kernbach, I. Kuksin, O. Kernbach, E. Kuksina, N. Mišković, S. Bogdan, T. Petrović, et al., subCULTron-Cultural Development as a Tool in Underwater Robotics, in: Proceedings of the Artificial Life and Intelligent Agents Symposium, Springer, 2016 , pp. $27-41$.

[177] M. Duarte, V. Costa, J. Gomes, T. Rodrigues, F. Silva, S. M. Oliveira, A. L. Christensen, Evolution of collective behaviors for a real swarm of aquatic surface robots, PloS one 11 (3).

[178] T. Schmickl, R. Thenius, C. Moslinger, J. Timmis, A. Tyrrell, M. Read, J. Hilder, J. Halloy, A. Campo, C. Stefanini, et al., Cocoro - the selfaware underwater swarm, in: Proceedings of the 5th IEEE Conference on Self-Adaptive and Self-Organizing Systems Workshops, 2011, pp. 120-126.

[179] G. K. Venayagamoorthy, Potentials and promises of computational intelligence for smart grids, Proceedings of the IEEE, 2009, pp. 1-6.

[180] W. Elmenreich, S. Schuster, Demand response by decentralized device control based on voltage level, in: Proceedings of the 7 th International 
Workshop on Self-Organizing Systems, Springer Verlag, 2013, pp. 186189.

[181] D. Steber, P. Bazan, R. German, Swarm - increasing households' internal 1845 pv consumption and offering primary control power with distributed batteries, in: Lecture Notes in Computer Science (including subseries Lecture Notes in Artificial Intelligence and Lecture Notes in Bioinformatics), Vol. 9424, Springer Verlag, 2015, pp. 3-11.

[182] Y. Huang, A. Brocco, P. Kuonen, M. Courant, B. Hirsbrunner, Smartgrid: A fully decentralized grid scheduling framework supported by swarm intelligence, in: Proceedings of the 7th International Conference on Grid and Cooperative Computing, 2008, pp. 160-168.

[183] B. Ramachandran, S. K. Srivastava, C. S. Edrington, D. A. Cartes, An intelligent auction scheme for smart grid market using a hybrid immune algorithm, IEEE Transactions on Industrial Electronics 58 (10) (2011) $4603-4612$.

[184] H. Kirchhoff, N. Kebir, K. Neumann, P. W. Heller, K. Strunz, Developing mutual success factors and their application to swarm electrification: microgrids with $100 \%$ renewable energies in the Global South and Germany, Journal of Cleaner Production 128 (2016) 190-200.

[185] G. Kyriakarakos, G. Papadakis, Multispecies Swarm Electrification for Rural Areas of the Developing World, Applied Sciences 9 (19) (2019) 3992.

[186] R. Muceka, T. Kukeera, Y. Alokore, K. Noara, S. Groh, Integrating a Solar PV System with a Household Based Backup Generator for Hybrid Swarm Electrification: A Case Study of Nigeria, in: Africa-EU Renewable Energy Research and Innovation Symposium 2018 (RERIS 2018), Springer, Cham, 2018, pp. 43-58. 
[187] M. Ghorbaniparvar, Survey on forced oscillations in power system, Journal of Modern Power Systems and Clean Energy 5 (2017) 671-682.

[188] J.-B. Mouret, K. Chatzilygeroudis, 20 years of reality gap: a few thoughts about simulators in evolutionary robotics, in: Proceedings of the Genetic and Evolutionary Computation Conference Companion, ACM, 2017, pp. 1121-1124, DOI: 10.1145/3067695.3082052.

[189] A. Sobe, W. Elmenreich, Smart microgrids: Overview and outlook, in: Proceedings of the ITG INFORMATIK Workshop on Smart Grids, Braunschweig, Germany, 2012.

[190] D. P. Stormont, Robot swarms for planetary exploration, in: Proceedings of the 4th International Conference and Exposition on Robotics for Challenging Situations and Environments, 2000, pp. 347-352.

[191] C. kwon Kang, Marsbee: Swarm of flapping wing flyers for enhanced Mars exploration, https://www.nasa.gov/directorates/spacetech/niac/ 2018_Phase_I_Phase_II/Marsbee_Swarm_of_Flapping_Wing_Flyers_ for_Enhanced_Mars_Exploration, [Online; accessed 9-July-2019] (2018).

[192] S. Yano, Kajima to develop automated construction machinery for building on Mars, Moon, https://asia.nikkei.com/TechScience/Tech/Kajima-to-develop-automated-constructionmachinery-for-building-on-Mars-moon, [Online; accessed 9-July2019] (2016).

[193] S. Caldwell, Nodes network \& operation demonstration satellite, https: //www.nasa.gov/centers/ames/engineering/projects/nodes.html, [Online; accessed 9-July-2019] (2015).

[194] G. Porter, Models of Proba-3 designs, http://www.esa.int/ spaceinimages/Images/2016/05/Models_of_Proba-3_designs, [Online; accessed 9-July-2019] (2016). 
[195] Kicksat, Kicksat: A tiny open source spacecraft project, https:// kicksat.github.io/, [Online; accessed 9-July-2019].

[196] S. D'Amico, M. Pavone, S. Saraf, A. Alhussien, T. Al-Saud, S. Buchman, R. Byer, C. Farhat, Miniaturized autonomous distributed space system for future science and exploration, in: Proceedings of the International Workshop on Satellite Constellations and Formation Flying, 2015, pp. $1-20$.

[197] M. Xining He, Swarm robotics: The future of medicine?, https: //medtechboston. medstro.com/blog/2015/10/06/swarm-roboticswhat-you-need-to-know-about-the-future-of-medicine/, [Online; accessed 15-September-2019] (2015).

[198] S. Hauert, S. N. Bhatia, Mechanisms of cooperation in cancer nanomedicine: Towards systems nanotechnology, Trends in Biotechnology 32 (9) (2014) 448-455.

[199] A. Purington, J. G. Taft, S. Sannon, N. N. Bazarova, S. H. Taylor, Alexa is my new bff: Social roles, user satisfaction, and personification of the amazon echo, in: Proceedings of the 2017 CHI Conference Extended Abstracts on Human Factors in Computing Systems, 2017, pp. 2853-2859.

[200] J. Aron, How innovative is apple's new voice assistant, siri?, Elsevier (2011).

[201] M. B. Hoy, Alexa, siri, cortana, and more: An introduction to voice assistants, Medical Reference Services Quarterly 37 (1) (2018) 81-88.

[202] S. Parikh, P. Atrey, Media-rich fake news detection: A survey, in: Proceedings of the IEEE Conference on Multimedia Information Processing and Retrieval, 2018, pp. 436-441.

[203] Q. Zhang, R. Segall, Web mining: A survey of current research, techniques, and software, International Journal of Information Technology \& Decision Making 7 (4) (2008) 683-720. 

service model for smart cities supported by internet of things, European Transactions on Telecommunications 25 (1) (2014) 81-93.

[205] C. Ferrer, The blockchain: A new framework for robotic swarm systems, in: K. Arai, R. Bhatia, S. Kapoor (Eds.), Proceedings of the Future Technologies Conference, Vol. 881 of Advances in Intelligent Systems and Computing, Springer, 2019.

[206] X.-S. Yang, S. Deb, Y.-X. Zhao, S. Fong, X. He, Swarm intelligence: Past, present and future, Soft Computing (2017) 1-11.

[207] I. Cohen, D. Corman, J. Davis, H. Khurana, P. J. Mosterman, S. L. Prasad Venkatesh, Strategic opportunities for 21st century cyber-physical systems, Tech. rep., NSF, Steering Committee for Foundations in Innovation for Cyber-Physical Systems (2012).

[208] A. Bagnato, R. K. Bíró, D. Bonino, C. Pastrone, W. Elmenreich, R. Reiners, M. Schranz, E. Arnautovic, Designing swarms of cyber-physical systems: The H2020 CPSwarm project, in: Proceedings of the Computing Frontiers Conference, 2017, pp. 305-312.

[209] J. Nagi, Symbiotic interaction between humans and robot swarms, Ph.D. thesis, Department of Informatics, University of Lugano, Switzerland (2016).

[210] A. Kolling, P. Walker, N. Chakraborty, K. Sycara, M. Lewis, Human interaction with robot swarms: A survey, IEEE Transactions on HumanMachine Systems 46 (1) (2016) 9-26.

[211] S. Jiang, R. C. Arkin, Mixed-initiative human-robot interaction: Definition, taxonomy, and survey, in: Proceedings of the IEEE International Conference on Systems, Man, and Cybernetics, 2015, pp. 954-961.

[212] A. Finzi, J. Cacace, R. Caccavale, V. Lippiello, Attentional multimodal interface for multi-drone search in the alps, in: Proceedings of the IEEE 
International Conference on Systems, Man, and Cybernetics, 2016, pp. $1178-1183$.

1955 [213] J. Nagi, A. Giusti, L. Gambardella, G. A. Di Caro, Human-swarm interaction using spatial gestures, in: Proceedings of the 27th IEEE/RSJ International Conference on Intelligent Robots and Systems, 2014, pp. $3834-3841$.

[214] G. Jones, N. Berthouze, R. Bielski, S. Julier, Towards a situated, multimodal interface for multiple UAV control, in: Proceedings of the IEEE International Conference on Robotics and Automation, 2010, pp. 17391744 . 


\section{CRediT author statement}

Melanie Schranz: Conceptualization, Investigation, Writing - Original Draft, Writing - Review \& Editing, Supervision, Project administration

Gianni A. Di Caro: Conceptualization, Investigation, Writing - Original Draft, Writing - Review \& Editing

Thomas Schmickl: Investigation, Writing - Review \& Editing

Wilfried Elmenreich: Investigation, Writing - Review \& Editing

Farshad Arvine: Investigation

Ahmet Sekercioglu: Conceptualization,

Micha Sende: Investigation, Writing - Review \& Editing 


\section{Declaration of Competing Interest}

The authors whose names are listed immediately below certify that they have NO affiliations with or involvement in any organization or entity with any financial interest (such as honoraria; educational grants; participation in speakers bureaus; membership, employment, consultancies, stock ownership, or other equity interest; and expert testimony or patent-licensing arrangements), or non-financial interest (such as personal or professional relationships, affiliations, knowledge or beliefs) in the subject matter or materials discussed in this manuscript. Author names:

Melanie Schranz

Gianni A. Di Caro

Thomas Schmickl

Wilfried Elmenreich

Farshad Arvine

Ahmet Sekercioglu

Micha Sende 